\title{
The Relative Contribution of Jumps to Total Price Variance
}

\author{
XIN HUANG \\ Duke University \\ George TAUCHEN \\ Duke University
}

\begin{abstract}
We examine tests for jumps based on recent asymptotic results; we interpret the tests as Hausman-type tests. Monte Carlo evidence suggests that the daily ratio $z$-statistic has appropriate size, good power, and good jump detection capabilities revealed by the confusion matrix comprised of jump classification probabilities. We identify a pitfall in applying the asymptotic approximation over an entire sample. Theoretical and Monte Carlo analysis indicates that microstructure noise biases the tests against detecting jumps, and that a simple lagging strategy corrects the bias. Empirical work documents evidence for jumps that account for $7 \%$ of stock market price variance.
\end{abstract}

KEYWORDS: bipower variation, quadratic variation, realized variance, stochastic volatility

Observers of financial markets have long noted that financial movements exhibit unusual behavior relative to what would be expected from the Gaussian distribution. There are too many small changes (inliers) and too many large changes (outliers). Clark (1973) is perhaps the first to formally investigate this behavior using econometric methods. He provides an explanation based on an embryonic form of the now familiar stochastic volatility model, as made formal in Taylor (1982, 1986), and studied extensively in the vast literature that follows [see

We thank Tim Bollerslev for many helpful discussions, and Ole Barndorff-Nielsen and Neil Shephard for graciously sharing the updates of their working papers with us. We also appreciate Neil Shephard, who gave detailed comments on earlier versions of this article and pointed out some important directions for the research, and Nour Meddahi, who also gave helpful comments on earlier versions and provided important suggestions for the study of the market microstructure noise. Two anonymous referees also provided extensive useful commentaries on an earlier draft. Address correspondence to George Tauchen, Department of Economics, Duke University, Box 90097, Durham, NC 27708, or E-mail: george.tauchen@duke.edu.

doi:10.1093/jjfinec/nbi025

Advance Access publication August 12, 2005

(C) The Author 2005. Published by Oxford University Press. All rights reserved. For permissions, please e-mail: journals.permissions@oupjournals.org. 
Shephard (2005)]. We now know that stochastic volatility can account for much of the dynamics of short-term financial price movements.

Modeling financial price changes in a way that implies the price series is the realization of a continuous-time diffusive process plays a central role in modern financial economics. The assumption of local continuous Gaussianity, among other things, simplifies the hedging calculations that underly modern derivatives pricing. Furthermore, as is well known, the superposition of multiple diffusive stochastic volatility processes can potentially accommodate the unusual dynamics mentioned just above; some examples are Gallant, Hsu, and Tauchen (1999) and Alizadeh, Brandt, and Diebold (2002), among others.

Although the diffusive models are of great analytical convenience, there remains the open issue of whether such models are empirically consistent with the extreme violent movements sometimes seen in financial price series. It is natural to ask whether jump diffusions, with discontinuous sample paths, provide a more appropriate empirical model for financial price series. Jump diffusions have a long and rich history in financial economics dating back at least to Merton (1976).

Jump diffusion models present two practical problems which some might view as nearly insurmountable while others might view as more minor nuisances. First, jump models are difficult to estimate, at least by simulation-based methods. The discontinuous sample paths create discontinuities in the econometric objective function that have to be accommodated by rounding out the corners, as in Andersen, Benzoni, and Lund (2002) and Chernov et al. (2003). Still, the nonlinear optimization remains difficult. It could well be the case that approximate likelihood methods based on Duffie, Pan, and Singleton (2000) or Ait-Sahalia (2004) entail a better-behaved econometric objective function, but that empirical work remains, to our knowledge, undone. Second, jumps introduce additional parameters into the derivatives pricing problem such as the price of jump risk and the price of intensity risk if the intensity is state dependent. These risk parameters are hard to interpret, not estimable in the time series alone, and difficult to pin down in the cross section. A case in point is Andersen, Benzoni, and Lund (2002), who estimate jump models and explore the implications of many of the possible branches for candidate values of these risk parameters. Thus it seems reasonable to attempt to preserve the simpler structure of purely diffusive models and thereby retain their convenience.

Chernov et al. (2003) provide empirical evidence that there are alternative, mildly nonlinear, purely diffusive models that provide, at the daily level, dynamics comparable to those of jump diffusions. However, they are unable to reach any firm conclusion on the empirical validity of one class of models over the other, and it is self-evident that higher frequency data are needed to provide more conclusive evidence on the empirical importance of jumps.

Barndorff-Nielsen and Shephard $(2004 b, 2006)$ develop a very powerful toolkit for detecting the presence of jumps in higher frequency financial time series. An appealing feature of their approach is that it does not require a fully observed state variable as in Ait-Sahalia (2002). Their basic idea is to compare two measures of 
variance, one of which includes the contribution of jumps, if any, to the total variance, while the second is robust to the jump contribution. A test of the statistical significance of the difference, suitably adjusted to improve asymptotic approximation, provides evidence on the presence of jumps. They implement the test on a high-frequency dataset of exchange rates, as do Andersen, Bollerslev, and Diebold (2004) on a broader set of assets; both articles adduce evidence that seemingly points to the presence of jumps on particular days of their datasets.

This article evaluates the properties of these newly developed jump detection tests. For the Monte Carlo data generating process we mainly use the single-factor log linear stochastic volatility model with jumps, which is the workhorse of applied econometrics on financial data. We supplement the analysis with consideration of the two-factor model of Chernov et al. (2003), a purely diffusive serious competitor to a jump diffusion. We examine size, power, and, in order to assess the tests' ability to identify correctly trading days on which a jump has occurred, the confusion matrix, whose elements are the probabilities of correct and incorrect classification. We also consider tests designed to address the question of whether an entire dataset is one generated from either a pure diffusion or jump diffusion model; to our knowledge, the full-sample-type tests have not been previously considered or analyzed.

Jump detection tests are constructed from very high frequency financial price data, which are potentially seriously contaminated by market microstructure noise. We examine theoretically the robustness of a generic jump test to microstructure noise of the sort commonly considered in the literature, and we consider the appropriateness of a correction strategy from Andersen, Bollerslev, and Diebold (2004). The theory delivers sharp predictions that are assessed by further Monte Carlo analysis.

Our empirical work focuses on five-minute returns on the S\&P Index, cash 1997-2002, and futures 1982-2002, with the objective of identifying the empirical importance of jumps as a source of price variance.

The remainder of this article is organized as follows. Section 1 sets up the notation and introduces the realized variance measures used for forming the jump test statistics. Section 2 reviews the joint asymptotic distribution of the realized measures. Section 3 summarizes the various jump detection tests. Section 4 reports on extensive Monte Carlo experiments that examine the behavior of the test statistics. Section 5 applies the tests to the S\&P 500 Index cash and futures data. Section 6 introduces market microstructure noise and examines both analytically and by Monte Carlo the effects of the noise on the jump tests. This section also examines an adjustment for the noise and reports the outcome of applying the adjusted tests to the S\&P 500 futures data. Finally, Section 7 contains concluding remarks.

\section{SETUP}

We consider a scalar log-price $p(t)$ evolving in continuous time as

$$
d p(t)=\mu(t) d t+\sigma(t) d w(t)+d L_{J}(t)
$$


where $\mu(t)$ and $\sigma(t)$ are the drift and instantaneous volatility, $w(t)$ is standardized Brownian motion, $L_{J}$ is a pure jump Lévy process with increments $L_{J}(t)-L_{J}(s)=\sum_{s \leq \tau \leq t} \kappa(\tau)$, and $\kappa(\tau)$ is the jump size. We adopt this notation from Basawa and Brockwell (1982). In this article we focus on a special class of the Lévy process called the compound Poisson process (CPP). It has constant jump intensity $\lambda$, and the jump size $k(t)$ is independent identically distributed (i.i.d.). Throughout, time is measured in daily units, and for integer $t$ we define the within-day geometric returns as

$$
r_{t, j}=p(t-1+j / M)-p(t-1+(j-1) / M), \quad j=1,2, \ldots, M,
$$

where $M$ is the sampling frequency.

Barndorff-Nielsen and Shephard (2004b) study general measures of realized within-day price variance, and two natural measures emerge from their work. The first is the now familiar realized variance,

$$
R V_{t}=\sum_{j=1}^{M} r_{t, j}^{2}
$$

and the other is the realized bipower variation,

$$
B V_{t}=\mu_{1}^{-2}\left(\frac{M}{M-1}\right) \sum_{j=2}^{M}\left|r_{t, j-1}\right|\left|r_{t, j}\right|=\frac{\pi}{2}\left(\frac{M}{M-1}\right) \sum_{j=2}^{M}\left|r_{t, j-1}\right|\left|r_{t, j}\right|,
$$

where

$$
\mu_{a}=\mathrm{E}\left(|Z|^{a}\right), \quad Z \sim N(0,1), a>0 .
$$

We use a slightly different notation that absorbs $\mu_{1}^{-2}$ into the definition of the bipower variation and thereby makes it directly comparable to the realized variance.

As noted in Andersen, Bollerslev, and Diebold (2002), the realized variance satisfies

$$
\lim _{M \rightarrow \infty} R V_{t}=\int_{t-1}^{t} \sigma^{2}(s) d s+\sum_{j=1}^{N_{t}} \kappa_{t, j}^{2}
$$

where $N_{t}$ is the number of jumps within day $t$ and $\kappa_{t, j}$ is the jump size. Thus the $R V_{t}$ is a consistent estimator of the integrated variance $\int_{t-1}^{t} \sigma^{2}(s) d s$ plus the jump contribution. On the other hand, the results of Barndorff-Nielsen and Shephard (2004b), along with extensions in Barndorff-Nielsen et al. (2005a, b), imply that under reasonable assumptions about the dynamics of Equation (1),

$$
\lim _{M \rightarrow \infty} B V_{t}=\int_{t-1}^{t} \sigma^{2}(s) d s
$$

Thus $B V_{t}$ provides a consistent estimator of the integrated variance unaffected by jumps. Evidently the difference $R V_{t}-B V_{t}$ is a consistent estimator of the pure 
jump contribution and, as emphasized by Barndorff-Nielsen and Shephard (2004b, 2006), can form the basis of a test for jumps. Andersen, Bollerslev, and Diebold (2004) use these results to generate evidence suggesting that there are too many large within-day movements in equity, fixed income, and foreign exchange prices to be consistent with the standard continuous-time stochastic volatility model with Markov volatility dynamics.

We also consider the relative jump measure

$$
R J_{t}=\frac{R V_{t}-B V_{t}}{R V_{t}},
$$

which is an indicator of the contribution (if any) of jumps to the total withinday variance of the process. An equivalent statistic, $-R J_{t}$, called the ratio statistic, is proposed and studied by Barndorff-Nielsen and Shephard (2006). We have a slight preference for the term relative jump, since $100 \cdot R J$ is a direct measure of the percentage contribution of jumps, if any, to total price variance.

Given a sample of $T$ days, we denote the total realized variance as

$$
R V_{1: T}=\sum_{t=1}^{T} R V_{t}
$$

and the total bipower variation as

$$
B V_{1: T}=\sum_{t=1}^{T} B V_{t}
$$

The corresponding relative jump measure is

$$
R J_{1: T}=\frac{R V_{1: T}-B V_{1: T}}{R V_{1: T}}
$$

\section{ASYMPTOTIC DISTRIBUTIONS}

Under the assumption of no jump and some other regularity conditions, Barndorff-Nielsen and Shephard (2006) first give the joint asymptotic distribution of $R V_{t}$ and $B V_{t}$, conditional on the volatility path, as $M \rightarrow \infty$,

$$
M^{\frac{1}{2}}\left[\int_{t-1}^{t} \sigma^{4}(s) d s\right]^{-\frac{1}{2}}\left(\begin{array}{l}
R V_{t}-\int_{t-1}^{t} \sigma^{2}(s) d s \\
B V_{t}-\int_{t-1}^{t} \sigma^{2}(s) d s
\end{array}\right) \stackrel{D}{\rightarrow} N\left(0,\left[\begin{array}{cc}
v_{q q} & v_{q b} \\
v_{q b} & v_{b b}
\end{array}\right]\right),
$$

where

$$
\left[\begin{array}{ll}
v_{q q} & v_{q b} \\
v_{q b} & v_{b b}
\end{array}\right]=\left[\begin{array}{cc}
\mu_{4}-\mu_{2}^{2} & 2\left(\mu_{3} \mu_{1}^{-1}-\mu_{2}\right) \\
2\left(\mu_{3} \mu_{1}^{-1}-\mu_{2}\right)\left(\mu_{1}^{-4}-1\right)+2\left(\mu_{1}^{-2}-1\right)
\end{array}\right],
$$

and using $\mu_{1}=\sqrt{ } \frac{2}{\pi}, \mu_{2}=1, \mu_{3}=2 \sqrt{ } \frac{2}{\pi}, \mu_{4}=3$, 


$$
\begin{aligned}
& v_{q q}=2 \\
& v_{q b}=2 \\
& v_{b b}=\left(\frac{\pi}{2}\right)^{2}+\pi-3 .
\end{aligned}
$$

The fact that asymptotically $v_{q b}=v_{q q}$ is no coincidence and reflects a situation exactly analogous to that of the Hausman (1978) test. Asymptotically the situation is one with Gaussian errors, and $R V_{t}$ is the most efficient estimate of the integrated variance $\int_{t-1}^{t} \sigma^{2}(s) d s$. The bipower variation is a less efficient estimator under the maintained assumption of no jumps, though it is also more robust. Thus, following the logic of the Hausman test:

Proposition 1 Under the maintained assumptions of no jumps, then asymptotically $R V_{t}-$ $B V_{t}$ is independent of $R V_{t}$ conditional on the volatility path, and thus $R J_{t}$ in Equation (2) is asymptotically the ratio of two conditionally independent random variables.

The proof is obvious by inspection. The above asymptotic distribution theory can be generalized considerably as in Barndorff-Nielsen, Graversen, Jacod, Podolskij, and Shephard (2005); Barndorff-Nielsen, Graversen, Jacod, and Shephard (2005); see Barndorff-Nielsen and Shephard (2005b) for a survey.

The relative jump measure, $R J_{t}$, has a natural notion of scale. If one is satisfied with this sense of scale, then there is no need to estimate the integrated quarticity $\int_{t-1}^{t} \sigma^{4}(s) d s$, as required for a standard deviation notion of scale.

To determine the scale of $R V_{t}-B V_{t}$ in units of conditional standard deviation, one needs to estimate the integrated quarticity $\int_{t-1}^{t} \sigma^{4}(s) d s$. Andersen, Bollerslev, and Diebold (2004) suggest using the jump-robust realized tri-power quarticity statistic, which is a special case of the multipower variations studied in Barndorff-Nielsen and Shephard (2004b),

$$
T P_{t}=M \mu_{4 / 3}^{-3}\left(\frac{M}{M-2}\right) \sum_{j=3}^{M}\left|r_{t, j-2}\right|^{4 / 3}\left|r_{t, j-1}\right|^{4 / 3}\left|r_{t, j}\right|^{4 / 3},
$$

and they note that

$$
T P_{t} \rightarrow \int_{t-1}^{t} \sigma^{4}(s) d s
$$

even in the presence of jumps. There is a scale normalizing constant $M$ in front of the summation because each absolute return is of order $\sqrt{\Delta t}$, so the product is of order $(\Delta t)^{2}$, and the summation $\Delta t . M$ is $\frac{1}{\Delta t}$, which cancels out the summation order, and the whole expression approaches a well-defined limit. As mentioned before, the value normalizing term is now $\mu_{4 / 3}^{-3}$, since each absolute return is raised to power $4 / 3$ and there are three such terms in one product. Notice that the power of each absolute return should be strictly less than two for the statistics to be robust to jumps. If it is equal to two, the statistics will behave just like $R V$, picking up both the jump and the continuous-time parts, and if it is greater than two, the 
whole expression will blow up to infinity because of the interaction between the scale normalizing constant and the jump component. Another estimator, based on Barndorff- Nielsen and Shephard (2004b), is the realized quad-power quarticity,

$$
Q P_{t}=M \mu_{1}^{-4}\left(\frac{M}{M-3}\right) \sum_{j=4}^{M}\left|r_{t, j-3}\right|\left|r_{t, j-2}\right|\left|r_{t, j-1}\right|\left|r_{t, j}\right| .
$$

Given a sample of $T$ days, the corresponding full-sample measures for TP and $Q P$ are

$$
\begin{aligned}
& T P_{1: T}=\sum_{t=1}^{T} T P_{t}, \\
& Q P_{1: T}=\sum_{t=1}^{T} Q P_{t} .
\end{aligned}
$$

\section{SOME JUMP TEST STATISTICS}

\subsection{Daily Statistics}

One strategy is to use the above theoretical results to compute a measure of extreme movements on a day-by-day basis and then inspect for days where the price movements appear abnormally large, which would be indicative of at least one jump that day. Based on Barndorff-Nielsen and Shephard's (2006) theoretical results, Andersen, Bollerslev, and Diebold (2004) use the time series

$$
z_{T P, t}=\frac{R V_{t}-B V_{t}}{\sqrt{\left(v_{b b}-v_{q q}\right) \frac{1}{M} T P_{t}}}
$$

to test for daily jumps. For each $t, z_{T P, t} \stackrel{D}{\rightarrow} N(0,1)$ as $M \rightarrow \infty$, on the assumption of no jumps. Thus the sequence $\left\{z_{T P, t}\right\}_{t=1}^{T}$ provides evidence on the daily occurrence of jumps in the price process. Another closely related measure is

$$
z_{Q P, t}=\frac{R V_{t}-B V_{t}}{\sqrt{\left(v_{b b}-v_{q q}\right) \frac{1}{M} Q P_{t}}},
$$

which uses the realized quad-power quarticity of Equation (4) in place of the realized tri-power quarticity of Equation (3) in the computation of the conditional scale of $R V_{t}-B V_{t}$.

Following Andersen, Bollerslev, Diebold, and Labys (2001, 2003); Andersen, Bollerslev, Diebold, and Ebens (2001); and Barndorff-Nielsen and Shephard (2005a), one might expect to be able to improve finite sample performance by basing the test statistics on the logarithm of the variation measures. In the case of Equation (5), the statistic is 


$$
z_{T P, l, t}=\frac{\log \left(R V_{t}\right)-\log \left(B V_{t}\right)}{\sqrt{\left(v_{b b}-v_{q q}\right) \frac{1}{M} \frac{T P_{t}}{B V_{t}^{2}}}},
$$

which is also used in Andersen, Bollerslev, and Diebold (2004). Another modification based on Barndorff-Nielsen and Shephard (2005a) entails the maximum adjustment

$$
z_{T P, l m, t}=\frac{\log \left(R V_{t}\right)-\log \left(B V_{t}\right)}{\sqrt{\left(v_{b b}-v_{q q}\right) \frac{1}{M} \max \left(1, \frac{T P_{t}}{B V_{t}^{2}}\right)}} .
$$

Analogous to the logarithmic adjustment to $z_{T P, t}$ we also have the statistic

$$
z_{Q P, l, t}=\frac{\log \left(R V_{t}\right)-\log \left(B V_{t}\right)}{\sqrt{\left(v_{b b}-v_{q q}\right) \frac{1}{M} \frac{Q P_{t}}{B V_{t}^{2}}}},
$$

and with the additional maximum adjustment, as used in Barndorff-Nielsen and Shephard (2004b), is

$$
z_{Q P, l m, t}=\frac{\log \left(R V_{t}\right)-\log \left(B V_{t}\right)}{\sqrt{\left(v_{b b}-v_{q q}\right) \frac{1}{M} \max \left(1, \frac{Q P_{t}}{B V_{t}^{2}}\right)}} .
$$

We also recall the measure of the relative jump (equivalent to negative of the ratio statistic):

$$
R J_{t}=\frac{R V_{t}-B V_{t}}{R V_{t}}
$$

and the statistics based on it are

$$
\begin{aligned}
& z_{T P, r, t}=\frac{R J_{t}}{\sqrt{\left(v_{b b}-v_{q q}\right) \frac{1}{M} \frac{T P_{t}}{B V_{t}^{2}}}}, \\
& z_{Q P, r, t}=\frac{R J_{t}}{\sqrt{\left(v_{b b}-v_{q q}\right) \frac{1}{M} \frac{Q P_{t}}{B V_{t}^{2}}}}, \\
& z_{T P, r m, t}=\frac{R J_{t}}{\sqrt{\left(v_{b b}-v_{q q}\right) \frac{1}{M} \max \left(1, \frac{T P_{t}}{B V_{t}^{2}}\right)}}, \\
& z_{Q P, r m, t}=\frac{R J_{t}}{\sqrt{\left(v_{b b}-v_{q q}\right) \frac{1}{M} \max \left(1, \frac{Q P_{t}}{B V_{t}^{2}}\right)}} .
\end{aligned}
$$

The $Q P$ versions of these statistics are equivalent to the ratio jump statistics of Barndorff-Nielsen and Shephard (2006). By visual inspection, one can see that the 
denominators of the log tests and the ratio tests are the same. In other words, the numerators of the second and third pairs of the jump statistics have identical asymptotic distributions conditional on the volatility path. The intuition is that the first-order Taylor expansions of the numerators of the log and the ratio test statistics around the asymptotic mean of $R V_{t}$ and $B V_{t}$, that is, the integrated variance $\int_{t-1}^{t} \sigma^{2}(s) d s$, are the same, thus the delta method generates the same asymptotic distribution.

In Section 4 we examine, among other things, the quality of the asymptotic normal approximation to each of these statistics under the null hypothesis of no jumps.

\subsection{Full-Sample Statistics}

We also consider the finite sample properties of the test statistics for jumps in a given sample. In this case, the test statistics are computed over the entire sample instead of on a day-by-day basis.

A natural asymptotically normal test statistic, following Andersen, Bollerslev, and Diebold (2004), is

$$
z_{T P, 1: T}=\frac{R V_{1: T}-B V_{1: T}}{\sqrt{\left(v_{b b}-v_{q q}\right) \frac{1}{M} T P_{1: T}}} .
$$

Another, based on the results of Barndorff-Nielsen and Shephard (2004b), is

$$
z_{Q P, 1: T}=\frac{R V_{1: T}-B V_{1: T}}{\sqrt{\left(v_{b b}-v_{q q}\right) \frac{1}{M} Q P_{1: T}}} .
$$

The log versions of these statistics are

$$
\begin{aligned}
& z_{T P, l, 1: T}=\frac{\log \left(R V_{1: T}\right)-\log \left(B V_{1: T}\right)}{\sqrt{\left(v_{b b}-v_{q q}\right) \frac{1}{M} \frac{T P_{1: T}}{B V_{1: T}}}}, \\
& z_{Q P, l: T}=\frac{\log \left(R V_{1: T}\right)-\log \left(B V_{1: T}\right)}{\sqrt{\left(v_{b b}-v_{q q}\right) \frac{1}{M} \frac{Q P_{1: T}}{B V_{1: T}^{2}}}} .
\end{aligned}
$$

With the additional maximum adjustment, the statistics become

$$
\begin{aligned}
& z_{T P, l m, 1: T}=\frac{\log \left(R V_{1: T}\right)-\log \left(B V_{1: T}\right)}{\sqrt{\left(v_{b b}-v_{q q}\right) \frac{1}{M} \max \left(\frac{1}{T}, \frac{T P_{1: T}}{B V_{1: T}^{2}}\right)}}, \\
& z_{Q P, l m, 1: T}=\frac{\log \left(R V_{1: T}\right)-\log \left(B V_{1: T}\right)}{\sqrt{\left(v_{b b}-v_{q q}\right) \frac{1}{M} \max \left(\frac{1}{T}, \frac{Q P_{1: T}}{B V_{1: T}^{2}}\right)}} .
\end{aligned}
$$

There are similar full-sample statistics based on $R J_{1: T}$ using $T P_{1: T}$ and $Q P_{1: T}$ as well: 


$$
\begin{aligned}
& z_{T P, r, 1: T}=\frac{R J_{1: T}}{\sqrt{\left(v_{b b}-v_{q q}\right) \frac{1}{M} \frac{T P_{1: T}}{B V_{1: T}^{2}}}}, \\
& z_{Q P, r, 1: T}=\frac{R J_{1: T}}{\sqrt{\left(v_{b b}-v_{q q}\right) \frac{1}{M} \frac{Q P_{1: T}}{B V_{1: T}^{2}}}}, \\
& z_{T P, r m, 1: T}=\frac{R J_{1: T}}{\sqrt{\left(v_{b b}-v_{q q}\right) \frac{1}{M} \max \left(\frac{1}{T}, \frac{T P_{1: T}}{B V_{1: T}^{2}}\right)}}, \\
& z_{Q P, r m, 1: T}=\frac{R J_{1: T}}{\sqrt{\left(v_{b b}-v_{q q}\right) \frac{1}{M} \max \left(\frac{1}{T}, \frac{Q P_{1: T}}{B V_{1: T}^{2}}\right)}} .
\end{aligned}
$$

A simple $t$-test on the relative jump measure is

$$
t_{R J, \text { classic }}=\frac{\frac{1}{T} \sum_{t=1}^{T} R J_{t}}{\sqrt{v_{\text {classic }}}},
$$

where $v_{\text {classic }}$ is the classical estimate of the variance of the mean computed under the assumption of no serial dependence. One can also form

$$
t_{R J, G M M}=\frac{\frac{1}{T} \sum_{t=1}^{T} R J_{t}}{\sqrt{v_{H A C}}},
$$

where $v_{\text {HAC }}$ is a HAC estimator of the variance of the mean. A bootstrap version is

$$
t_{R J, \text { boot }}=\frac{\frac{1}{T} \sum_{t=1}^{T} R J_{t}}{\sqrt{v_{\text {boot }}}},
$$

where $v_{\text {boot }}$ is a bootstrap estimate of the variance of the mean. Finally, by bootstrapping $t_{R J \text {,classic }}$ one can get a bootstrap confidence interval $\left(t_{\text {low }}, t_{\text {up }}\right)$ for the $t$-statistic and form a test that way. We have not computed these more complicated $t$-statistics because the evidence and theory suggest that $R J_{t}$ are essentially serially uncorrelated, if the jump part follows the compound Poisson process. Interested readers are referred to Gonçalves and Meddahi (2005) for an in-depth study of the test statistics based on the bootstrap variance of the realized variance measures.

\section{MONTE CARLO ANALYSIS}

\subsection{Setup}

We analyze the behavior of the various tests above under two classes of models for the $\log$ price process $p_{t}$. The first is a stochastic volatility jump diffusion model of the form

SV1FJ : $\quad d p(t)=\mu d t+\exp \left[\beta_{0}+\beta_{1} v(t)\right] d w_{p}(t)+d L_{J}(t)$

$$
d v(t)=\alpha_{v} v(t) d t+d w_{v}(t)
$$


where the $w^{\prime}$ s are standard Brownian motions, $\operatorname{corr}\left(d w_{p}, d w_{v}\right)=\rho$ is the leverage correlation, $v(t)$ is a stochastic volatility factor, $L_{J}(t)$ is a compound Poisson process with constant jump intensity $\lambda$ and random jump size distributed as $N\left(0, \sigma_{j m p}^{2}\right)$. The model SV1FJ has one stochastic volatility factor and a jump; it has been widely studied. Barndorff-Nielsen and Shephard (2004b) term this a stochastic volatility model with rare jumps. A special case without a jump term is

$$
\begin{aligned}
\text { SV1F }: & d p(t)=\mu d t+\exp \left[\beta_{0}+\beta_{1} v(t)\right] d w_{p}(t) \\
d v(t) & =\alpha_{v} v(t) d t+d w_{v}(t) .
\end{aligned}
$$

We also follow Chernov et al. (2003) and consider a two-factor stochastic volatility model,

$$
\mathbf{S V 2 F : ~} \begin{aligned}
d p(t) & =\mu d t+\operatorname{s}-\exp \left[\beta_{0}+\beta_{1} v_{1}(t)+\beta_{2} v_{2}(t)\right] d w_{p}(t) \\
d v_{1}(t) & =\alpha_{v 1} v_{1}(t) d t+d w_{v 1}(t) \\
d v_{2}(t) & =\alpha_{v 2} v_{2}(t) d t+\left[1+\beta_{v 2} v_{2}(t)\right] d w_{v 2}(t),
\end{aligned}
$$

where $v_{1}(t)$ and $v_{2}(t)$ are stochastic volatility factors. The process $v_{1}(t)$ is a standard Gaussian process, while $v_{2}(t)$ exhibits a feedback term in the diffusion function. The feedback is found to be important in Chernov et al. (2003). The function s-exp means the usual exponential function with a polynomial function splined in at very high values of its argument to ensure that the system of Equation (24) with $\beta_{v 2} \neq 0$ satisfies the growth conditions for a solution to exist and for the Euler scheme to work. The s-exp function is considered more fully below. The leverage correlations are $\operatorname{corr}\left(d w_{p}, d w_{v 1}\right)=\rho_{1}$ and $\operatorname{corr}\left(d w_{p}, d w_{v 2}\right)=\rho_{2}$.

The SV2F model has continuous sample paths, but it can generate quite rugged appearance price series via the volatility feedback and the exponential function, which is splined only at relatively high values of its argument. One of our objectives is to examine whether the various jump test statistics will falsely signal jumps under a process with continuous sample paths.

Table 1 shows the parameter settings used in the simulations for the SV1FJ model, with, of course, SV1F representing the null hypothesis. The parameter

Table 1 Experimental design for SV1FJ models.

\begin{tabular}{lc}
\hline$\mu$ & 0.030 \\
$\beta_{0}$ & 0.000 \\
$\beta_{1}$ & 0.125 \\
$\alpha_{v}$ & $\{-0.137 \mathrm{e}-2,-0.100,-1.386\}$ \\
$\rho$ & -0.620 \\
$\lambda$ & $\{0.014,0.058,0.082,0.118\}$ \\
& $\{0.50,1.00,1.50,2.00\}$ \\
$\sigma_{\text {jmp }}$ & $\{0.00 \ldots 2.50$ by 0.50$\}$ \\
ntick & 60 \\
nstep & 390 \\
\hline
\end{tabular}


Table 2 Experimental design for SV2F model.

\begin{tabular}{lc}
\hline$\mu$ & \\
$\beta_{0}$ & 0.030 \\
$\beta_{1}$ & -1.200 \\
$\beta_{2}$ & 0.040 \\
$\alpha_{v 1}$ & 1.500 \\
$\alpha_{v 2}$ & $-0.137 \mathrm{e}-2$ \\
$\beta_{v 2}$ & -1.386 \\
$\rho_{p, v 1}$ & 0.250 \\
$\rho_{p, v 2}$ & -0.300 \\
\hline
\end{tabular}

values are based on the empirical results reported in Andersen, Benzoni, and Lund (2002), Andersen, Bollerslev, and Diebold (2004), and Chernov et al. (2003). The three values of the volatility mean reversion parameter $\alpha_{v}$ represent very slow mean reversion, with a half-life of two years $(2 \times 252$ trading days $)$, medium mean reversion, with a half-life just over one week, and very strong mean reversion with a half-life of 0.50 days. The very slow and very strong values for the mean reversion parameter are based on Chernov et al. (2003), while the medium value is based on Andersen, Benzoni, and Lund (2002). There are four values for the jump intensity $(\lambda)$. The smallest jump intensity is estimated by Andersen, Benzoni, and Lund (2002) for the daily S\&P 500 cash index, while the other three come from Andersen, Bollerslev, and Diebold (2004) for the high-frequency data on the deutschemark/dollar (DM/\$) spot market, S\&P 500 index futures, and U.S. Treasury-bond futures markets. The parameter $\sigma_{j m p}$ varies over a range that includes Andersen, Benzoni, and Lund's (2002) estimate of about $1.50 \%$. The value of the leverage parameter is from Andersen, Benzoni, and Lund (2002), though our experiments suggest that the findings are not very sensitive to this parameter. The scaling parameters, that is, the $\beta$ 's, are selected on the basis of the studies and some experiments guided by plots of simulated data. For computational reasons, the above-mentioned empirical studies use different normalizations, different timing conventions, and they simulated arithmetic, not geometric returns, so it is difficult to match up the values exactly to those previously estimated.

The simulation details are as follows: the basic unit of time is one day throughout. Simulations of the diffusion parts of the SV1FJ and SV2F models are generated using the basic Euler scheme with an increment of one second per tick on the Euler clock. We first simulate the log price level, then compute the 1-minute, 3-minute, 5-minute, and 30-minute geometric returns by taking the difference of the corresponding log price levels, with the objective to see how sampling frequency affects the properties of the test statistics. In order to make results comparable across sampling intervals, it is important to simulate a single Brownian motion at a very fine time interval, use the Euler scheme to solve for a simulation from the nonlinear model, and then sample that series at coarser 
intervals. The jump component is simulated by drawing the jump times from the exponential distribution and the jump size from $\mathrm{N}\left(0, \sigma_{j m p}^{2}\right)$.

The simulation of the SV2F model requires some special attention to satisfy the growth conditions [Kloeden and Platen (1992: 128)] of Ito's theorem. Following Chernov et al. (2003), we spline smoothly to the far right-hand side of the exponential function the growth function itself, so the model satisfies the regularity conditions by construction. The knot point for the spline is a value of the argument of the exponential that implies a $100 \%$ annualized volatility, which is unlikely to occur in the normal U.S. financial markets. Inspection of very long simulations revealed the spline has no essential effect, except for attenuating the influences of a very few really large values of the argument.

Figure 1 and Figure 2 show the simulated realizations of length 10,000 days (about 40 years) at the daily frequency from the SV1F and SV1FJ models, with medium mean reversion, $\sigma_{j m p}=1.50$ and $\lambda=0.014$ in the latter case. The daily returns seen in the second panel appear reasonable for a financial time series. Likewise, Figure 3 shows a simulation at the daily frequency from the SV2F model, which looks very similar to the plots of daily returns found in the financial econometrics literature.
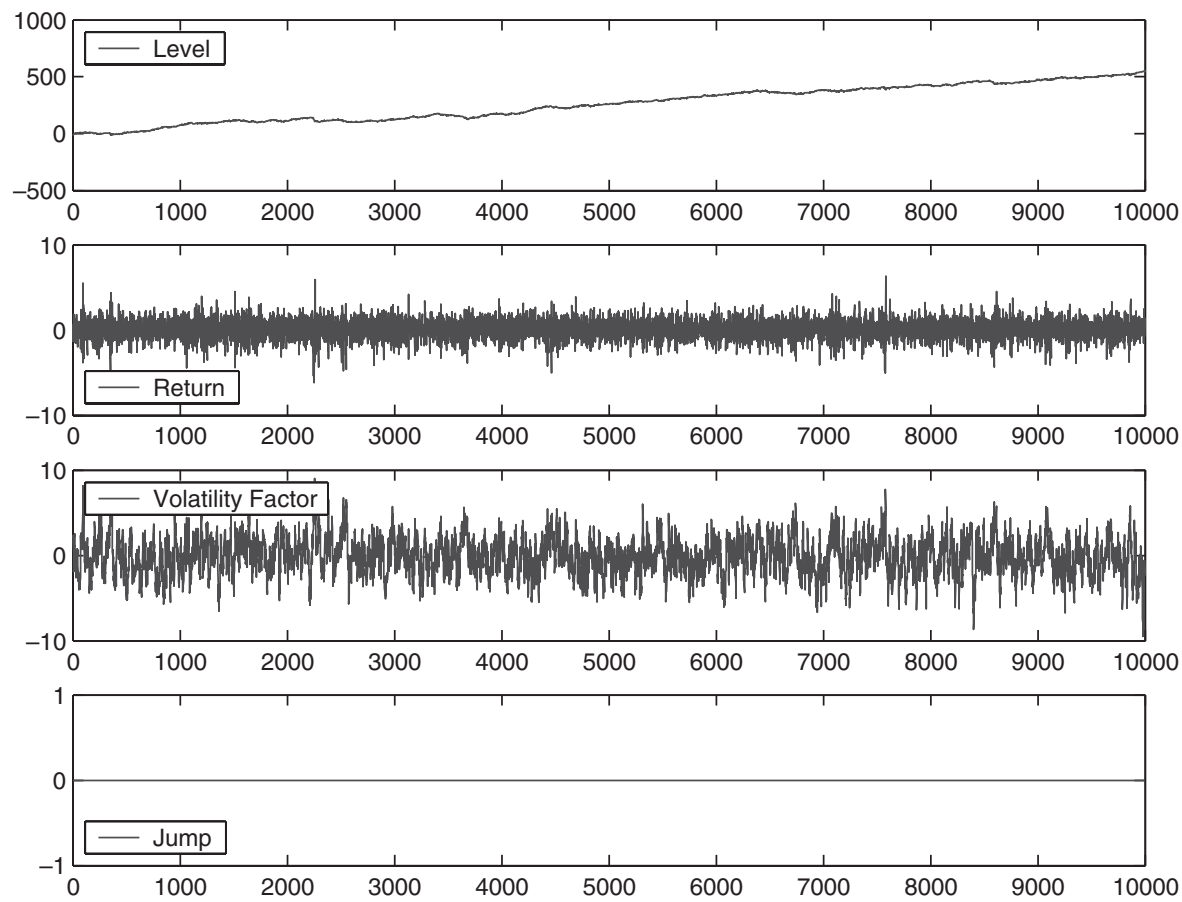

Figure 1 Simulated realization from the SV1F model, daily frequency, for 10,000 days, using fiveminute returns under medium mean reversion $\left(\alpha_{v}=-0.100\right)$. 

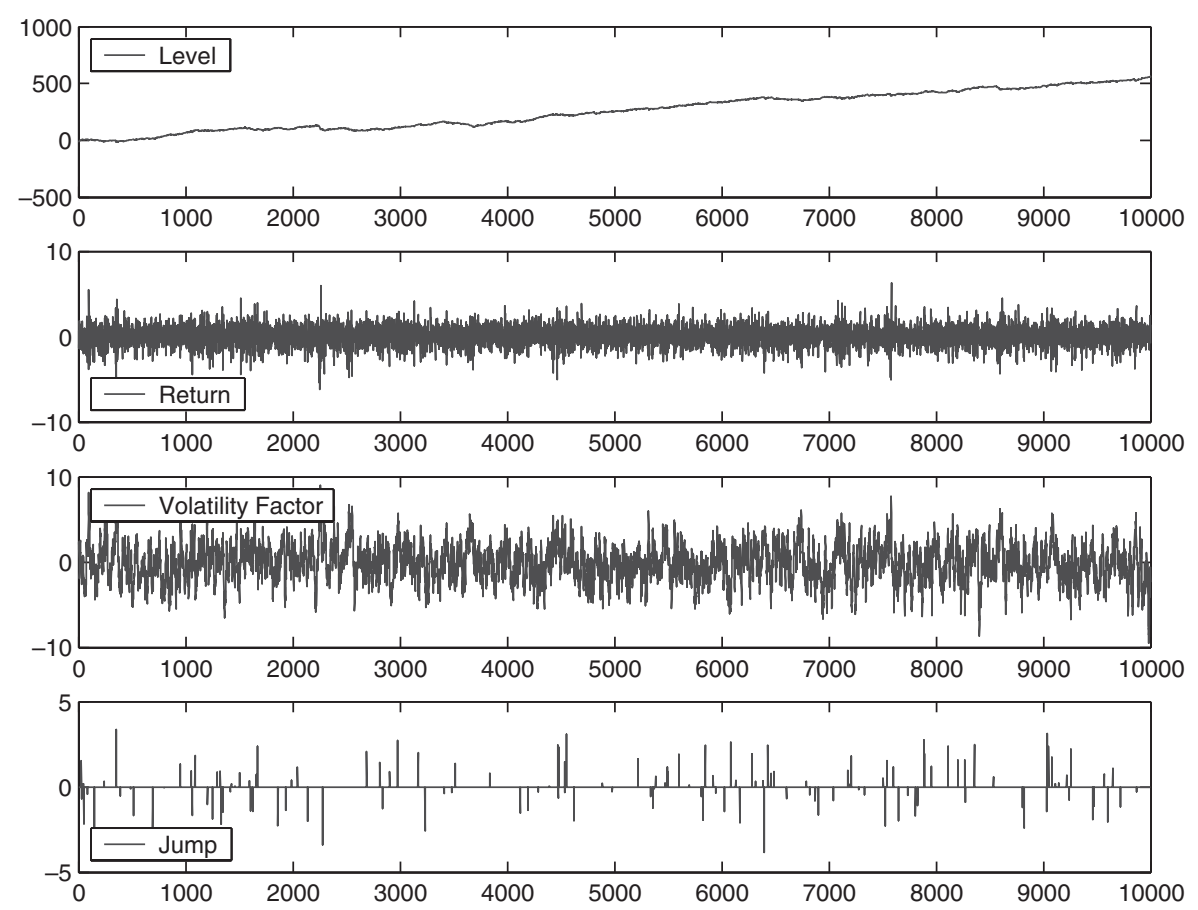

Figure 2 Simulated realization from the SV1FJ model, daily frequency, for 10,000 days, using five-minute returns under medium mean reversion $\left(\alpha_{v}=-0.100\right), \sigma_{j m p}=1.50$, and $\lambda=0.014$.

\subsection{Monte Carlo Findings}

4.2.1 Daily Statistics. We first consider the characteristics of the daily statistics computed over long simulated realizations, $\left\{z_{T P, t}\right\}_{t=1}^{N}$ and $\left\{z_{Q P, t}\right\}_{t=1}^{N}$, of length $N=45,000$.

4.2.1.1 Size. Figure 4 shows QQ plots of the raw, log-max, and ratio-max adjusted versions of $z_{T P, t}$ defined in Equations (5), (7), and (9). Since the log and ratio adjusted versions are similar, they are not shown here. The data generation process is the null case in Table $1, \sigma_{j m p}=0.00$, with medium mean reversion $\left(\alpha_{v}=\right.$ $-0.100)$ and 5-minute returns. Since large values of the $z$-statistics discredit the null hypothesis of no jumps, we are only interested in the right-hand tail. As is clear from the figures, the raw statistic has a size distortion toward overrejecting in the range 2.00 to 3.00 , about the 0.99 to 0.999 significance level, which is the usual range considered for these daily z-statistics. However, the log transformation and the statistic based on $R J$ correct the size distortions, except perhaps in the extreme right-hand tail. Although the boundary in the maximum adjustment is hit a lot, the QQ plots do not appear to change much when we add the maximum adjustment. A similar situation shows up when we study the full-sample statis- 

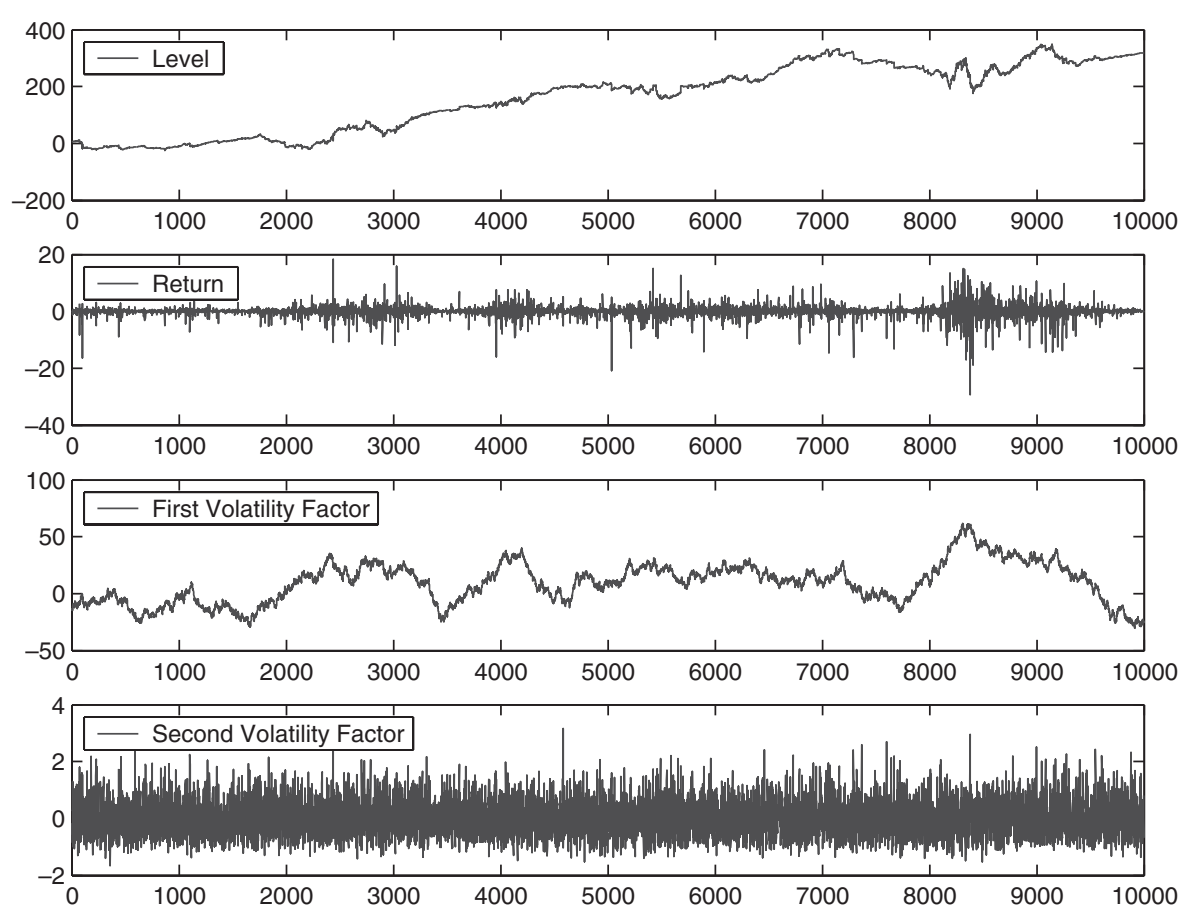

Figure 3 Simulated realization from the SV2F model, daily frequency, for 10,000 days, using fiveminute returns.

tics. Apparently the deviations from the boundary are not serious, thus they do not affect the value of the $z$-statistics significantly.

As seen from Figure 5, the raw statistic $z_{Q P, t}$ has the same size problem and the effects of the $\log$, ratio, and max adjustments are the same as with $z_{T P, t}$. It appears that the choice between $T P_{t}$ and $Q P_{t}$ does not matter in any important way for the estimation of $\int_{t-1}^{t} \sigma^{4}(s) d s$. Interestingly, Figure 6 indicates that the relative jump statistic, $R J_{t}$, is very well approximated by a Gaussian distribution.

With the exception of the $z_{T P, r m, t}$ statistic, the sampling frequency has a significant impact on the size. As the sampling frequency decreases, that is, the sampling interval increases, the actual sizes of all statistics except $z_{T P, r m, t}$ increase above the Monte Carlo confidence band, which can be seen in Figure 7 for the medium mean reversion case. The behavior of the size for the slow mean reversion and the fast cases are the same, so they are not shown here.

Figure 8 shows a simulation of length 1400 days of the five versions of $z_{T P, t}$ statistics under the null of no jumps and $\alpha_{v}=-0.100$. We choose 1400 because that is very close to the sample size for the cash index in the empirical application below. The size problem is apparent in the top panel as is the correction due to the $\log$ adjustment and ratio adjustment. Note that $z_{T P, r m, t}$ in the bottom panel appears to have the best size property among the five statistics. 

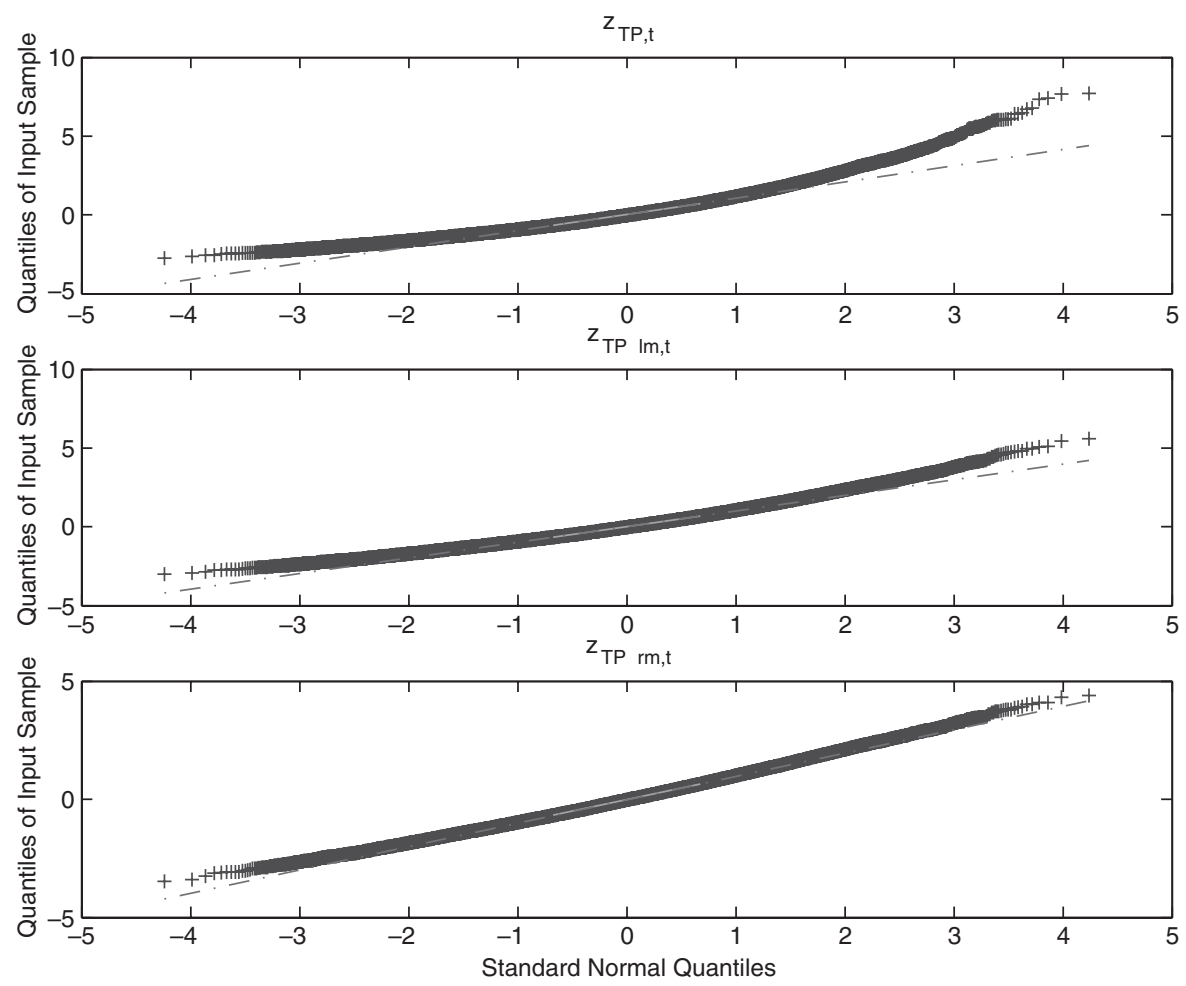

Figure 4 QQ plots, $z_{T P}$ daily statistics, for 45,000 days, using five-minute returns under medium mean reversion $\left(\alpha_{v}=-0.100\right)$.

4.2.1.2 Jump Detection. For evidence on the ability of the tests to detect jumps under the SV1FJ setting, Figure 9 shows a simulation of the versions of $z_{T P, t}$ under the same conditions as in Figure 8, except with $\sigma_{j m p}=1.50$ and $\lambda=0.014$. As is clear, the statistics do a very good job of picking out the jumps.

To see how the parameter settings affect the detection ability of the test statistics, we report the confusion matrix under different parameter settings. The matrix consists of four cells: the upper left cell is the proportion of the statistic smaller than the $99 \%$ standard Gaussian critical value among days without jumps, the upper right cell is the proportion greater than the $99 \%$ critical value among the no-jump days, the lower left cell is the proportion smaller than the $99 \%$ critical value among the days that jump occur, and the lower right one is the proportion greater than the $99 \%$ critical value among the jump days. The diagonal elements of this matrix represent the ability of the test statistic to tell correctly whether or not there is a jump in a particular day, and the off-diagonal represent the proportion of days when the statistic signals the wrong answer. The row sums of this matrix equal unity. Under the asymptotic theory presented in the previous sections, we expect the $(1,1)$ element of this matrix to be close to $99 \%$, as the 

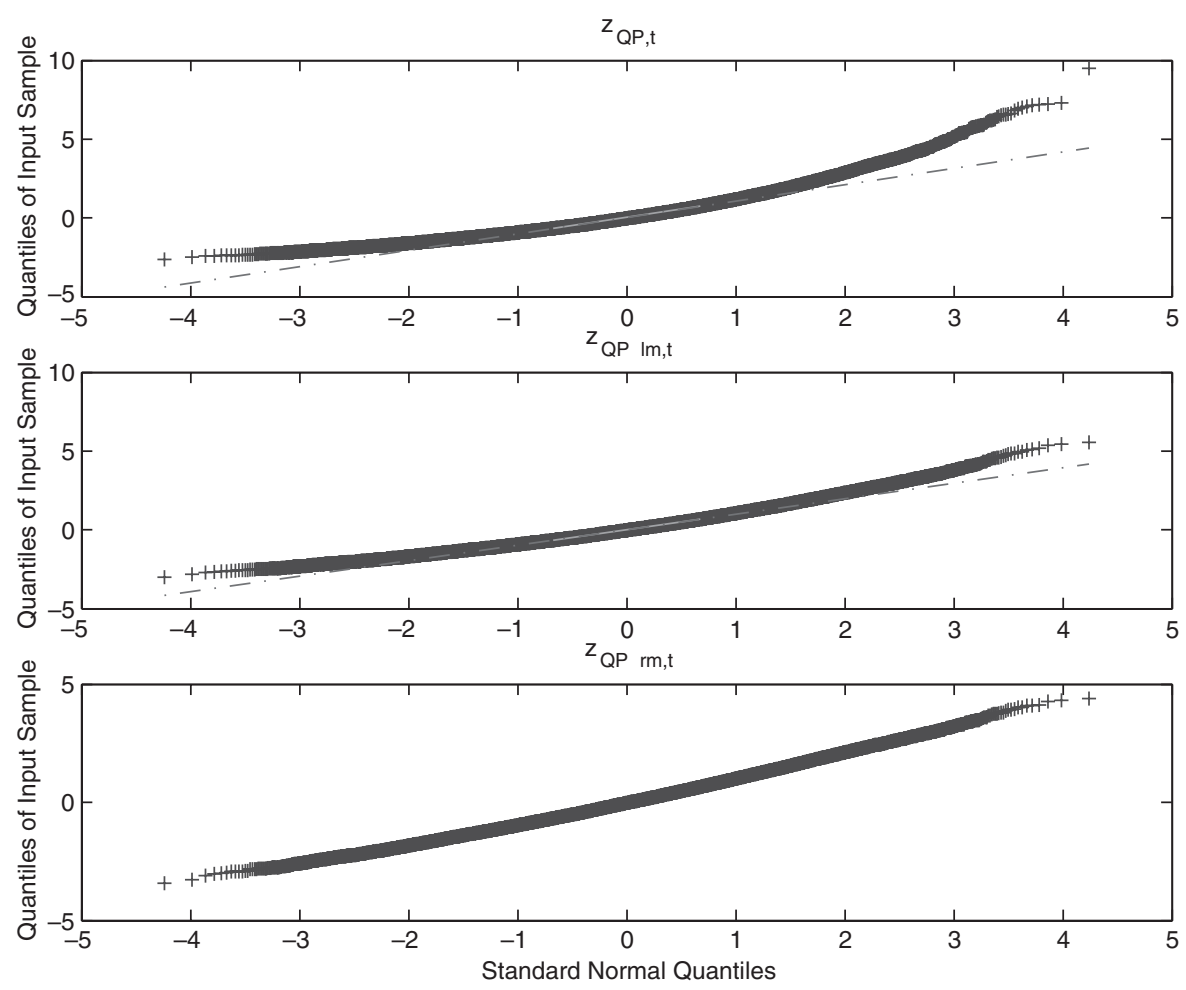

Figure 5 QQ plots, $z_{Q P}$ daily statistics, for 45,000 days, using five-minute returns under medium mean reversion $\left(\alpha_{v}=-0.100\right)$.

sampling interval goes to zero; however, the $(2,2)$ element of this matrix needs not approach unity, as explained in subsection 4.2.1.3.

Table 3 contains the confusion matrices for the three test statistics$z_{T P, t}, z_{T P, l m, t}$, and $z_{T P, r m, t}$-over different jump intensities and sampling frequencies under medium mean reversion $\left(\alpha_{v}=-0.100\right)$ and jump size 1.50 . The mean reversion does not significantly impact the daily statistics, and the jump size positively affects the rejection frequency in the expected manner, so we do not report variations in these two parameters here. By comparing the first rows of the matrices for the three test statistics, we can see that $z_{T P, t}$ tends to signal more false jumps when there is no jump in a particular day, and the problem becomes more severe with longer sampling intervals. On the other hand, $z_{T P, r m, t}$ is most robust, with a false rejection rate close to the nominal size of $1 \%$. By examining the second rows of the matrices, we see that as the sampling interval increases from 1 minute to 30 minutes, the test statistics signal fewer instances of jumps when jumps occur because of the time averaging effect. An increase in jump intensity, $\lambda$, has a positive effect on the detection rate. The reason is that the statistics detect jumps through the 

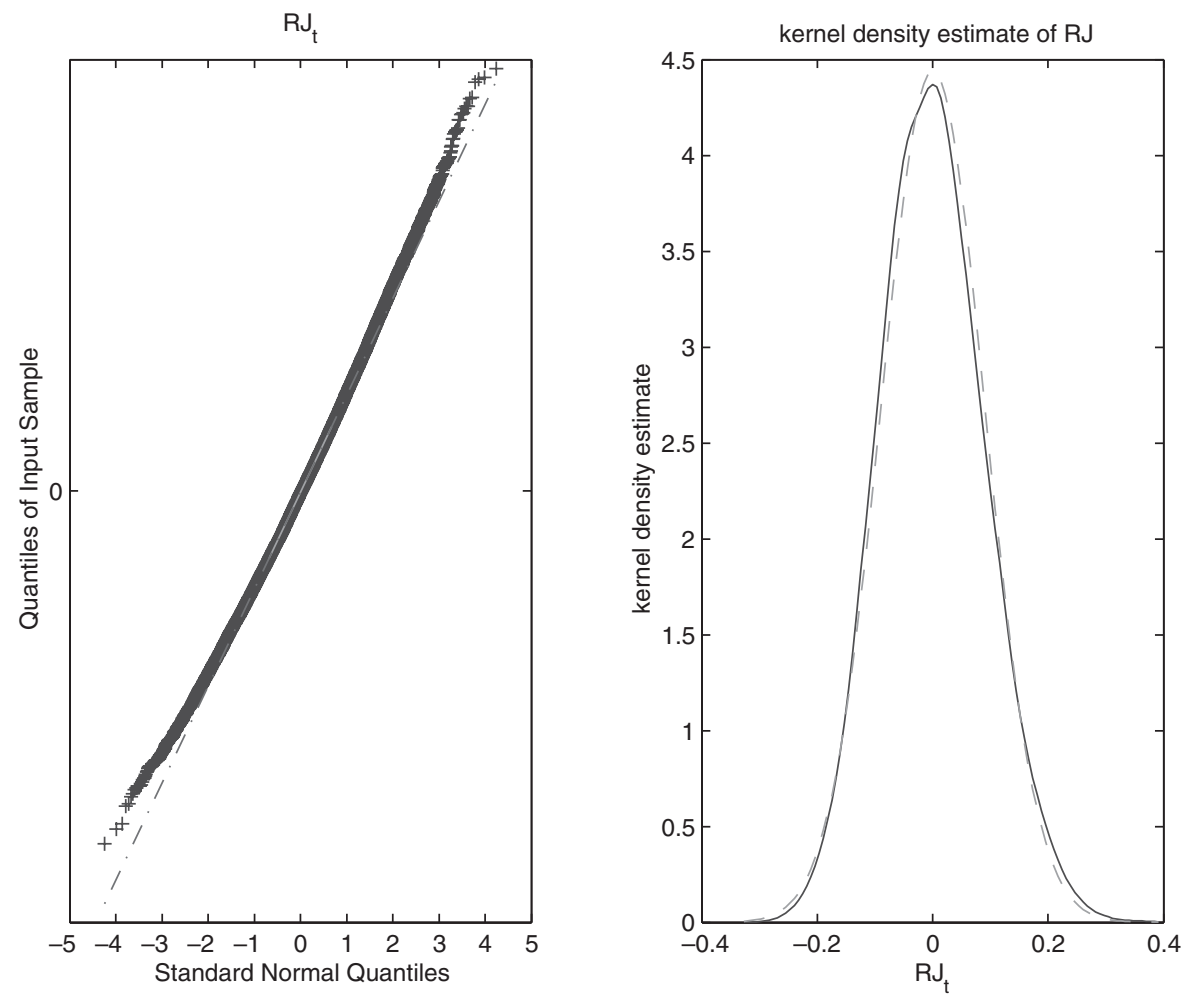

Figure 6 QQ plot and kernel density plot, $R J_{t}$ daily statistic for 45,000 days, using five-minute returns under medium mean reversion $\left(\alpha_{v}=-0.100\right)$.

proportion of the total price variation attributable to jumps. When $\lambda$ increases, the expected number of jumps per day increases. This increases the expected accumulated jump contribution per day, making it more likely that the statistics detect the jumps.

4.2.1.3 Power. The above jump detection results are most likely to be important for daily application purposes, but from the statistical viewpoint, we are also interested in the power of the tests. Table 4 reports the power of the three test statistics, $z_{T P, t}, z_{T P, l m, t}$, and $z_{T P, r m, t}$, over different jump intensities and jump sizes under medium mean reversion using five-minute simulated returns. Jump intensity and jump size have positive effects on power. These results are intuitive. Table 5 shows the power property of $z_{T P, r m, t}$ over different jump intensities, jump sizes, and sampling frequencies. Just like the effect on the jump detection rate, the sampling frequency impacts power positively. So combining the results in size, jump detection rate, and power, we can see that, in the absence of market microstructure noise, for lower sampling frequency, the statistics not only neglect true jumps when there are jumps (lower jump detection rate and lower power), 

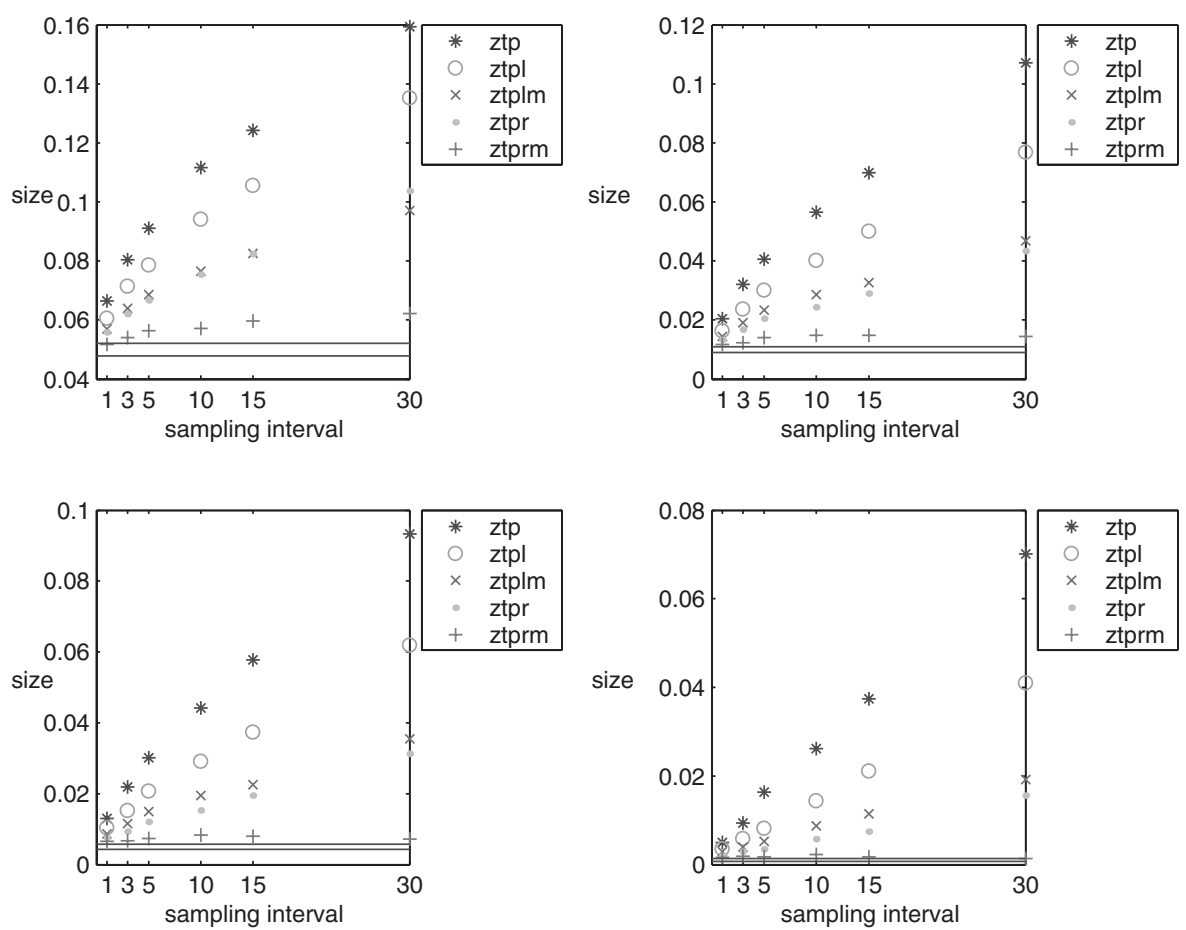

Figure 7 The size of the jump statistics over different sampling intervals under the medium mean reversion $\left(\alpha_{v}=-0.100\right)$. The nominal size is 0.05 for the upper-left subplot, 0.005 for the lower-left subplot, 0.01 for the upper-right subplot, and 0.001 for the lower-right subplot. The two horizontal lines are the $95 \%$ Monte Carlo confidence bands corresponding to the nominal size. The sample size is 45,000 days and the return horizon is five minutes.

but also signal more false jumps when there is no jump (larger size). So highfrequency data are necessary for jump detection when we use these types of statistics.

In addition to the effects of different parameters on the test statistics, an important phenomenon is apparent in these two tables. The test is inconsistent; that is, its power will not approach one as the sample size goes to infinity. The reason is that, for any finite jump intensity, the underlying jump-diffusion process does not have jumps every day. The time interval between two jumps is exponentially distributed, and the probability of having no jump for a day is $e^{-\lambda}$, which is not zero for any $\lambda<\infty$. Even when $\lambda$ is as high as two (unlikely to occur in the empirical data), such a probability is still 0.135 . Since the test statistics signal jumps for only a very small portion of the no-jump days, their power, defined as the proportion of signaled jump days over the whole sample, will not approach one.

4.2.1.4 The SV2F Model. The above results show that the test statistics have excellent size property under the SV1F(J) model. In contrast, however, Figure 10 shows a simulation of the $z_{T P, t}$ statistics under the SV2F model described above. 


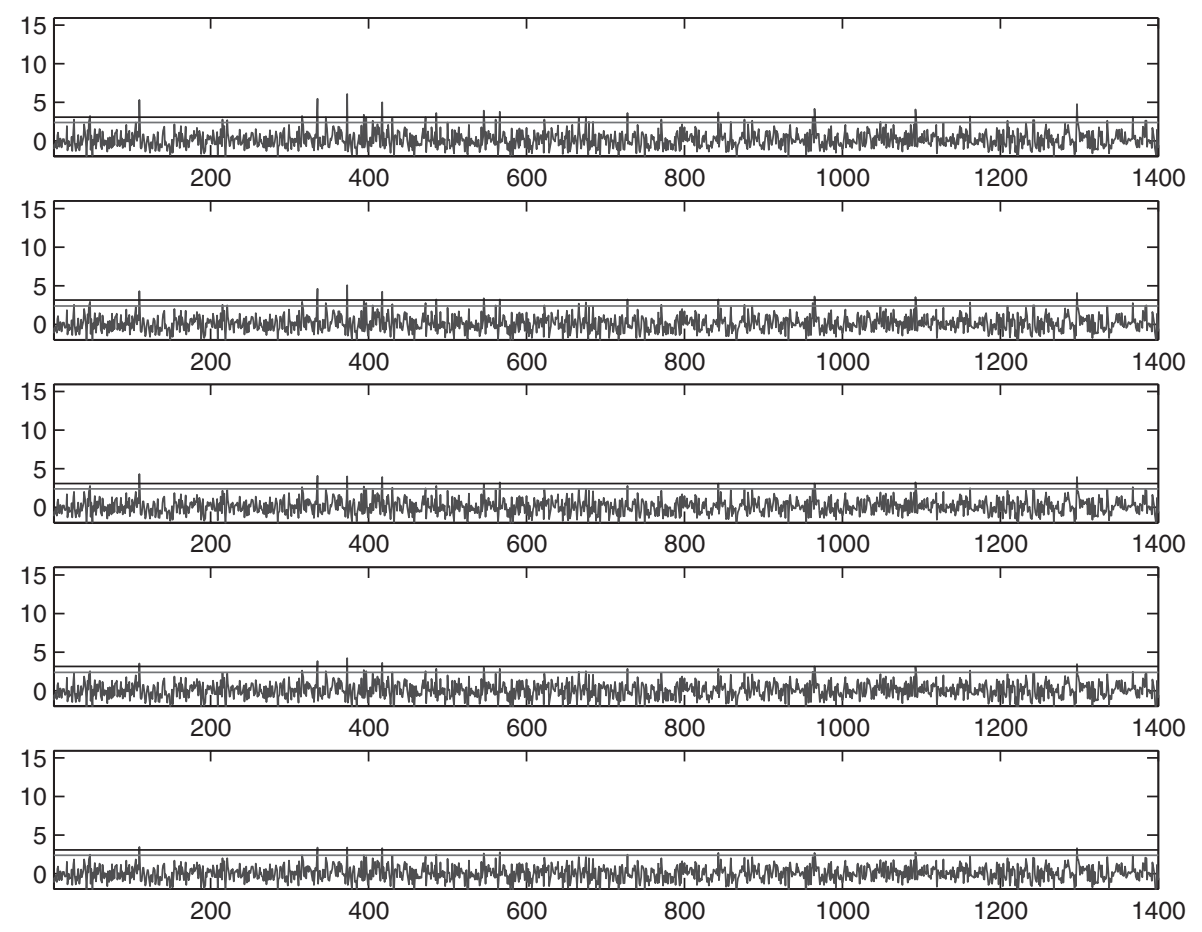

Figure 8 Simulated time series of $z_{T P, t}$ 's under the SV1F model. The five panels show simulations of the basic statistic, the log version, the max-log version, the ratio version, and the max-ratio version for $\sigma_{j m p}=0$ under medium mean reversion. The horizontal lines are the upper 0.99 and 0.999 critical values of the standard Gaussian distribution. The sample size is 1400 days and the return horizon is five minutes.

The underlying model does not contain jumps, though the simulation indicates detection of spurious jumps. The figure suggests the test statistics have incorrect size. Table 6 for five-minute returns further illustrates this point. The $95 \%$ confidence interval for the nominal size of $5 \%$ over 45,000 simulation days is [0.04799, $0.05201]$, for the size of $1 \%$ is $[0.00908,0.01092]$, for the size of $0.5 \%$ is [0.00435, 0.00565], and for the size of $0.1 \%$ is [0.00071, 0.00129]. However, all the empirical sizes are outside these confidence intervals, although the size of the $z_{T P, r m, t}$ is least affected. The finding of overrejection is in contrast to that of Barndorff-Nielsen and Shephard (2006), who utilize the superposition of two square-root [Cox, Ingersoll, and Ress (CIR)] volatility processes. It suggests that the curvature of the volatility functions influences the properties of the test statistics somewhat, although the size distortions in Table 6 are not very large from a practical point of view.

4.2.2 Monte Carlo Assessment of Full-Sample Statistics. We now consider the test of the null hypothesis that a given dataset has been generated from a data generating process without jumps versus the alternative of one with jumps. This 


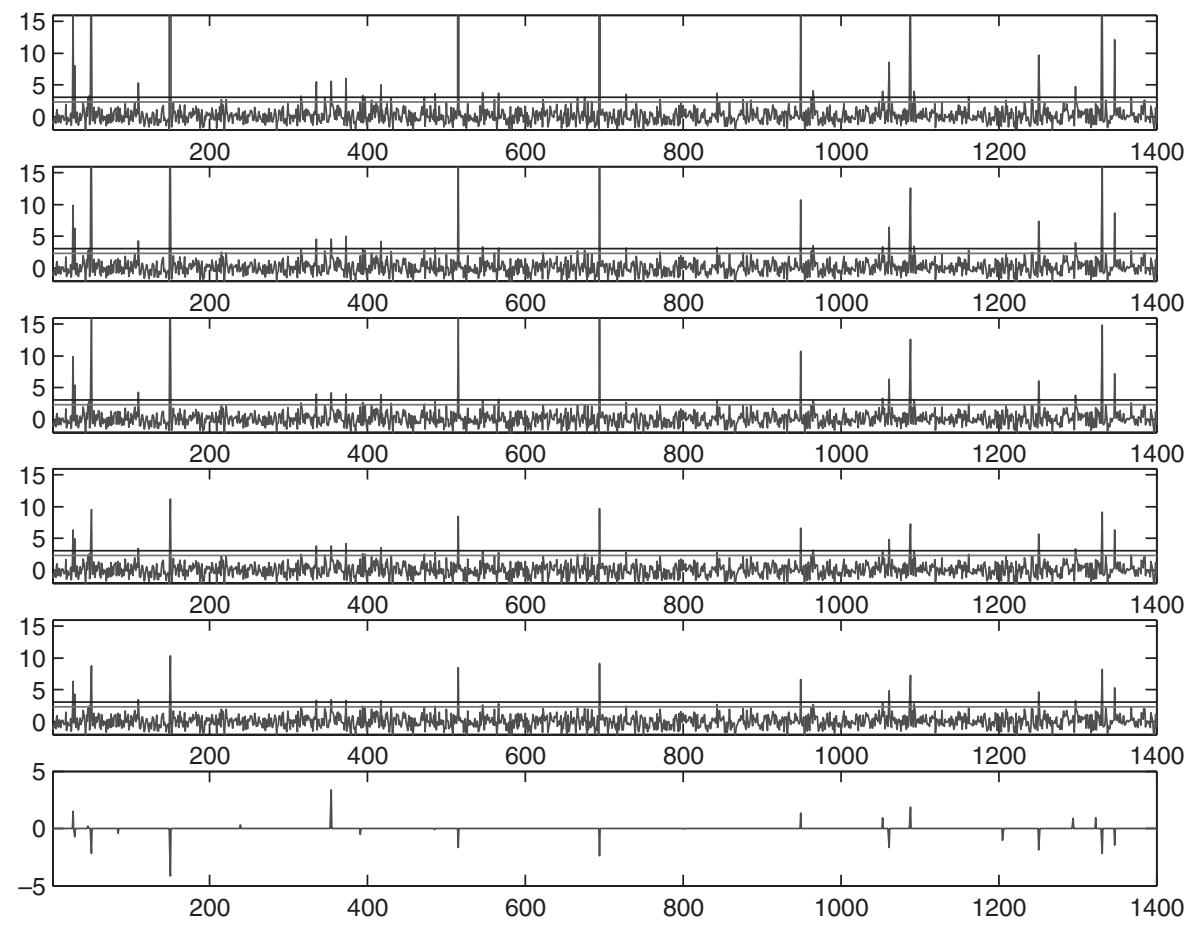

Figure 9 Simulated time series of $z_{T P, t}$ 's under the SV1FJ model. The five panels show simulations of the basic statistic, the log version, the max-log version, the ratio version, and the maxratio version for $\sigma_{j m p}=0$ and $\lambda=0.014$ under medium mean reversion. The horizontal lines are the upper 0.99 and 0.999 critical values of the standard Gaussian distribution. The bottom panel shows the jumps in the simulated realization. The sample size is 1400 days and the return horizon is five minutes.

question is different from the one just considered, where the issue was whether or not a jump occurred on a particular day. Tests of this null hypothesis are based on the full sample, rather than being computed day by day. The candidate test statistics are displayed in Equations (10)-(22).

Perhaps the most interesting tests are those based $z_{T P, 1: T}$ and $z_{Q P, 1: T}$ and their transforms as defined in Equations (10)-(19). Table 7 shows the rejection frequencies under the one-factor SV1FJ and two factor SV2F models for 1000 repetitions of a time series with 1400 days. The tests appear to have excellent size and power properties under the SV1FJ model with the strong and medium mean reversions, while, as might be expected, the power of each test under the slow mean reversion is somewhat lower because the diffusive part of the model accounts for a relatively larger share of the variance. However, the tests seem to have incorrect size under the SV2F model for this sample size.

The finding that, under the SV2F model, the full-sample statistics incorrectly reject the null of no jump more often than the daily statistics is notable, but needs 
Table 3 Confusion matrices.

\begin{tabular}{|c|c|c|c|c|c|c|c|c|c|c|}
\hline \multirow[b]{2}{*}{ Interval } & & & \multicolumn{2}{|c|}{$\lambda=0.014$} & \multicolumn{2}{|c|}{$\lambda=0.118$} & \multicolumn{2}{|c|}{$\lambda=1.000$} & \multicolumn{2}{|c|}{$\lambda=2.000$} \\
\hline & & & (NJ) & (J) & (NJ) & (J) & (NJ) & (J) & $(\mathrm{NJ})$ & (J) \\
\hline \multirow[t]{6}{*}{1 minute } & \multirow[t]{2}{*}{$z_{T P, t}$} & (NJ) & 0.980 & 0.020 & 0.981 & 0.019 & 0.989 & 0.011 & 0.995 & 0.005 \\
\hline & & $(\mathrm{J})$ & 0.205 & 0.795 & 0.178 & 0.822 & 0.132 & 0.868 & 0.080 & 0.920 \\
\hline & \multirow[t]{2}{*}{$z_{T P, l m, t}$} & (NJ) & 0.986 & 0.014 & 0.986 & 0.014 & 0.993 & 0.007 & 0.996 & 0.004 \\
\hline & & (J) & 0.208 & 0.792 & 0.184 & 0.816 & 0.136 & 0.864 & 0.084 & 0.916 \\
\hline & \multirow[t]{2}{*}{$z_{T P, r m, t}$} & (NJ) & 0.988 & 0.012 & 0.989 & 0.011 & 0.994 & 0.006 & 0.997 & 0.003 \\
\hline & & $(\mathrm{J})$ & 0.214 & 0.786 & 0.187 & 0.813 & 0.139 & 0.861 & 0.086 & 0.914 \\
\hline \multirow[t]{6}{*}{3 minutes } & \multirow[t]{2}{*}{$z_{T P, t}$} & (NJ) & 0.968 & 0.032 & 0.969 & 0.031 & 0.982 & 0.018 & 0.992 & 0.008 \\
\hline & & (J) & 0.273 & 0.727 & 0.261 & 0.739 & 0.198 & 0.802 & 0.125 & 0.875 \\
\hline & \multirow[t]{2}{*}{$z_{T P, l m, t}$} & $(\mathrm{NJ})$ & 0.981 & 0.019 & 0.982 & 0.018 & 0.989 & 0.011 & 0.996 & 0.004 \\
\hline & & $(\mathrm{J})$ & 0.286 & 0.714 & 0.274 & 0.726 & 0.208 & 0.792 & 0.132 & 0.868 \\
\hline & \multirow[t]{2}{*}{$z_{T P, r m, t}$} & (NJ) & 0.988 & 0.012 & 0.988 & 0.012 & 0.993 & 0.007 & 0.997 & 0.003 \\
\hline & & (J) & 0.292 & 0.708 & 0.285 & 0.715 & 0.219 & 0.781 & 0.141 & 0.859 \\
\hline \multirow[t]{6}{*}{5 minutes } & \multirow[t]{2}{*}{$z_{T P, t}$} & (NJ) & 0.960 & 0.040 & 0.963 & 0.037 & 0.980 & 0.020 & 0.991 & 0.009 \\
\hline & & (J) & 0.302 & 0.698 & 0.314 & 0.686 & 0.239 & 0.761 & 0.160 & 0.840 \\
\hline & \multirow[t]{2}{*}{$z_{T P, l m, t}$} & (NJ) & 0.977 & 0.023 & 0.978 & 0.022 & 0.988 & 0.012 & 0.994 & 0.006 \\
\hline & & (J) & 0.347 & 0.653 & 0.337 & 0.663 & 0.257 & 0.743 & 0.175 & 0.825 \\
\hline & \multirow{2}{*}{$z_{T P, r m, t}$} & (NJ) & 0.986 & 0.014 & 0.987 & 0.013 & 0.993 & 0.007 & 0.997 & 0.003 \\
\hline & & (J) & 0.360 & 0.640 & 0.358 & 0.642 & 0.274 & 0.726 & 0.191 & 0.809 \\
\hline \multirow[t]{6}{*}{30 minutes } & \multirow[t]{2}{*}{$z_{T P, t}$} & (NJ) & 0.894 & 0.106 & 0.899 & 0.101 & 0.943 & 0.057 & 0.974 & 0.026 \\
\hline & & (J) & 0.558 & 0.442 & 0.543 & 0.457 & 0.489 & 0.511 & 0.432 & 0.568 \\
\hline & \multirow[t]{2}{*}{$z_{T P, l m, t}$} & $(\mathrm{NJ})$ & 0.954 & 0.047 & 0.957 & 0.043 & 0.975 & 0.025 & 0.988 & 0.012 \\
\hline & & $(\mathrm{J})$ & 0.620 & 0.380 & 0.641 & 0.359 & 0.590 & 0.410 & 0.540 & 0.460 \\
\hline & \multirow[t]{2}{*}{$z_{T P, r m, t}$} & (NJ) & 0.986 & 0.014 & 0.987 & 0.013 & 0.992 & 0.008 & 0.996 & 0.004 \\
\hline & & (J) & 0.744 & 0.257 & 0.749 & 0.251 & 0.706 & 0.294 & 0.671 & 0.329 \\
\hline
\end{tabular}

Fixed parameters: level of significance $=0.01, \sigma_{j m p}=1.50, \alpha_{v}=-0.10$. The columns represent the jump days signaled by the statistics and the rows are the actual days on which jumps occur in the simulation.

to be interpreted properly. The realized bipower variation and realized variance, though consistent, are not unbiased for the integrated variance. That is, for any finite sampling frequency $M$, it can be the case that, if the expectations exist,

$$
\mathrm{E}\left(R V_{t}-B V_{t}\right)=B(M) \neq 0,
$$

although one expects $B(M)$ to be rather small. However, relative to the daily statistics, the full-sample statistics increase the time span without shrinking the sampling interval, so in effect the small bias gets inflated to $T \cdot B(M)$ and the $z$-statistics can become very large. Therefore the larger the sample size, the more apparent the difference between $R V_{1: T}$ and $B V_{1: T}$, and hence the greater the rejection frequency of the full-sample $z$-statistics.

There are other strategies for forming full-sample test statistics. For example, one can add the sum of squared daily z-statistics and treat it as a chi-square 
Table 4 Power for different jump statistics.

\begin{tabular}{lcccccc}
\hline & \multicolumn{7}{c}{$\sigma_{j m p}$} \\
\cline { 2 - 7 } & $\lambda$ & 0.5 & 1.0 & 1.5 & 2.0 & 2.5 \\
\hline $\mathrm{z}_{\mathrm{TP}, \mathrm{t}}$ & 0.500 & 0.14718 & 0.25651 & 0.30640 & 0.33291 & 0.34789 \\
& 1.000 & 0.24060 & 0.42038 & 0.49578 & 0.53458 & 0.55727 \\
& 1.500 & 0.31969 & 0.54704 & 0.63264 & 0.67322 & 0.69687 \\
& 2.000 & 0.39227 & 0.64382 & 0.73042 & 0.77042 & 0.79278 \\
$\mathrm{z}_{\mathrm{TP}, \mathrm{Im}, \mathrm{t}}$ & 0.500 & 0.12260 & 0.23538 & 0.28713 & 0.31613 & 0.33236 \\
& 1.000 & 0.21082 & 0.39747 & 0.47767 & 0.51931 & 0.54389 \\
& 1.500 & 0.28569 & 0.52367 & 0.61540 & 0.65993 & 0.68567 \\
& 2.000 & 0.35569 & 0.62182 & 0.71567 & 0.75967 & 0.78309 \\
$z_{T P, r m, t}$ & 0.500 & 0.10493 & 0.21949 & 0.27309 & 0.30480 & 0.32216 \\
& 1.000 & 0.18669 & 0.37782 & 0.46264 & 0.50660 & 0.53282 \\
& 1.500 & 0.25767 & 0.50193 & 0.59967 & 0.64758 & 0.67436 \\
& 2.000 & 0.32433 & 0.59936 & 0.70044 & 0.74709 & 0.77291 \\
\hline
\end{tabular}

Fixed parameters: level of significance $=0.010, \alpha_{v}=-0.100$, and five-minute returns.

Table 5 Power of $z_{T P, r m, t}$ for different sampling frequencies.

\begin{tabular}{lcccccc}
\hline \multirow{5}{*}{ 1 minute } & \multicolumn{7}{c}{$\sigma_{\text {jmp }}$} \\
\cline { 2 - 7 } & $\lambda$ & 0.5 & 1.0 & 1.5 & 2.0 & 2.5 \\
\hline \multirow{5}{*}{3 minutes } & 0.500 & 0.20420 & 0.29744 & 0.33033 & 0.34693 & 0.35651 \\
& 1.000 & 0.36213 & 0.50140 & 0.54920 & 0.57158 & 0.58462 \\
& 1.500 & 0.48840 & 0.64236 & 0.69351 & 0.71711 & 0.72993 \\
& 2.000 & 0.58822 & 0.74833 & 0.79222 & 0.81340 & 0.82502 \\
5 minutes & 0.500 & 0.13633 & 0.24873 & 0.29624 & 0.32167 & 0.33607 \\
& 1.000 & 0.24687 & 0.42813 & 0.49971 & 0.53400 & 0.55520 \\
& 1.500 & 0.33969 & 0.56329 & 0.64049 & 0.67773 & 0.69902 \\
& 2.000 & 0.42362 & 0.66578 & 0.74324 & 0.77909 & 0.79756 \\
& 0.500 & 0.10493 & 0.21949 & 0.27309 & 0.30480 & 0.32216 \\
& 1.000 & 0.18669 & 0.37782 & 0.46264 & 0.50660 & 0.53282 \\
& 1.500 & 0.25767 & 0.50193 & 0.59967 & 0.64758 & 0.67436 \\
& 2.000 & 0.32433 & 0.59936 & 0.70044 & 0.74709 & 0.77291 \\
& 0.500 & 0.02500 & 0.06642 & 0.11384 & 0.15609 & 0.18787 \\
& 1.000 & 0.03451 & 0.10747 & 0.19082 & 0.25720 & 0.30787 \\
& 1.500 & 0.04102 & 0.13958 & 0.24260 & 0.32618 & 0.38818 \\
& 2.000 & 0.04893 & 0.16556 & 0.28342 & 0.37276 & 0.43624 \\
\hline
\end{tabular}

Fixed parameters: level of significance $=0.010, \alpha_{v}=-0.100$.

random variable. Another is to compute over the stimulated sample of length $T$ the proportion of days on which the individual z-statistics are statistically significant at some given level $\alpha$, and then form the usual pivotal statistic 


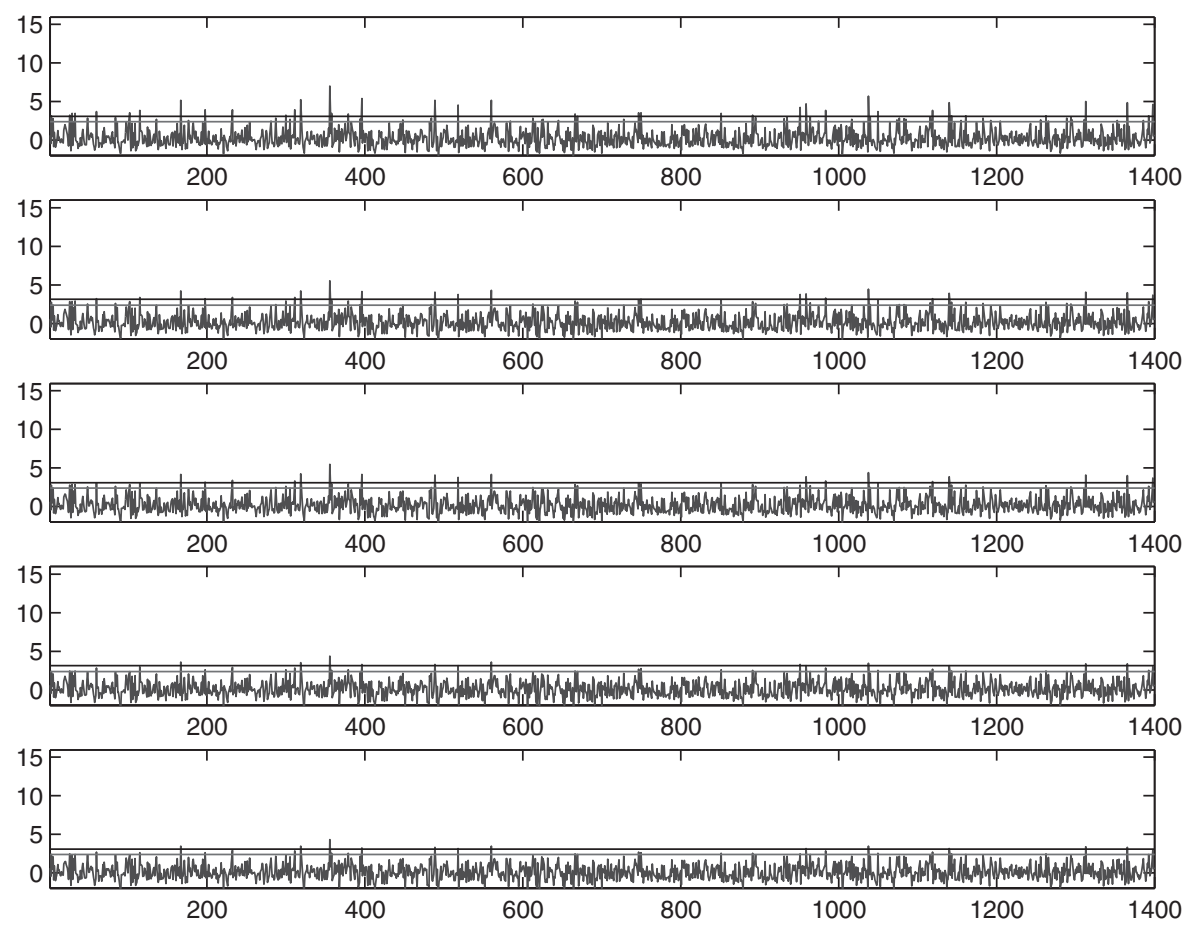

Figure 10 Simulated time series of $z_{T P, t}$ 's under the SV2F model. The five panels show simulations of the basic statistic, the log version, the max-log version, the ratio version, and the max-ratio version. The horizontal lines are the upper 0.99 and 0.999 critical values of the standard Gaussian distribution. The sample size is 1400 days and the return horizon is five minutes.

Table 6 SV2F model results, daily.

\begin{tabular}{lcccc}
\hline & size $(5 \%)$ & size $(1 \%)$ & size $(0.5 \%)$ & size $(0.1 \%)$ \\
\hline$z_{T P, t}$ & 0.13589 & 0.07616 & 0.06209 & 0.04056 \\
$z_{T P, l, t}$ & 0.12024 & 0.05931 & 0.04544 & 0.02664 \\
$z_{T P, l m, t}$ & 0.11709 & 0.05642 & 0.04284 & 0.02436 \\
$z_{T P, r, t}$ & 0.10171 & 0.04167 & 0.03016 & 0.01471 \\
$z_{T P, r m, t}$ & 0.09829 & 0.03867 & 0.02747 & 0.01276 \\
\hline
\end{tabular}

Fixed parameters: five-minute returns; see Table 2 for the others.

based on the Gaussian approximation to the binomial. We experimented with these strategies and always found the same conclusions as just described above. When replicated over many samples of length $T$, the tests can incorrectly overreject the null of no jumps, because a tiny daily bias gets inflated by the factor $T$.

Full-sample tests can potentially become consistent tests for jumps with proper size, if a way can be found to eliminate the bias. There are possible 
Table 7 Rejection frequencies, full sample.

\begin{tabular}{|c|c|c|c|c|c|c|c|c|c|c|c|c|}
\hline \multirow[b]{2}{*}{$\sigma_{j m p}$} & \multicolumn{2}{|c|}{$z_{T P}$} & \multicolumn{2}{|c|}{$z_{T P, l m}$} & \multicolumn{2}{|c|}{$z_{T P, r m}$} & \multicolumn{2}{|c|}{$z_{Q P}$} & \multicolumn{2}{|c|}{$z_{Q P, l m}$} & \multicolumn{2}{|c|}{$z_{Q P, r m}$} \\
\hline & 0.01 & 0.05 & 0.01 & 0.05 & 0.01 & 0.05 & 0.01 & 0.05 & 0.01 & 0.05 & 0.01 & 0.05 \\
\hline \multicolumn{13}{|c|}{ SV1FJ: $\alpha_{v}=-1.3860$, fast mean revision } \\
\hline 0.00 & 0.015 & 0.056 & 0.015 & 0.055 & 0.015 & 0.055 & 0.015 & 0.057 & 0.015 & 0.057 & 0.015 & 0.056 \\
\hline 0.50 & 0.106 & 0.306 & 0.106 & 0.306 & 0.105 & 0.303 & 0.106 & 0.307 & 0.106 & 0.307 & 0.105 & 0.303 \\
\hline 1.00 & 885 & 0.948 & 0.882 & 0.947 & 0.882 & 0.947 & 0.885 & 0.948 & 0.883 & 0.947 & 0.883 & 0.947 \\
\hline 1.50 & 994 & 0.995 & 0.994 & 0.995 & 0.994 & 0.995 & 0.994 & 0.995 & 0.994 & 0.995 & 0.994 & 0.995 \\
\hline 2.00 & 000 & 1.000 & 1.000 & 1.000 & 1.00 & 1.000 & 1.000 & 1.000 & 1.000 & 1.000 & 1.000 & 1.000 \\
\hline 2.50 & 1.000 & 1.000 & 1.000 & 1.000 & 1.000 & 1.000 & 1.000 & 1.000 & 1.000 & 1.000 & 1.000 & 1.000 \\
\hline \multicolumn{13}{|c|}{ SV1FJ: $\alpha_{v}=-0.100$, medium mean reversion } \\
\hline 0.00 & 0.009 & 0.059 & 0.008 & 0.059 & 0.008 & 0.058 & 0.009 & 0.059 & 0.008 & 0.059 & 0.008 & 0.058 \\
\hline 0.50 & 0.067 & 0.209 & 0.064 & 0.209 & 0.064 & 0.206 & .068 & 0.212 & 0.0 & 0.210 & 0.064 & 0.206 \\
\hline 1.00 & 743 & 0.878 & 0.740 & 0.878 & 0.739 & 0.878 & 0.743 & 0.878 & 0.742 & 0.878 & 0.739 & 0.878 \\
\hline 1.50 & 983 & 0.990 & 0.983 & 0.990 & 0.983 & 0.990 & 0.983 & 0.990 & 0.983 & 0.990 & 0.983 & 0.990 \\
\hline 2.00 & 999 & 0.999 & 0.999 & 0.999 & 0.999 & 0.999 & 0.999 & 0.999 & 0.999 & 0.999 & 0.999 & 0.999 \\
\hline 2.50 & 1.000 & 1.000 & 1.000 & 1.000 & 1.000 & 1.000 & 1.000 & 1.000 & 1.000 & 1.000 & 1.000 & 1.000 \\
\hline \multicolumn{13}{|c|}{ SV1FJ: $\alpha_{v}=-0.137 e-02$, slow mean reversion } \\
\hline 0.00 & 0.004 & 0.032 & 0.003 & 0.031 & 0.003 & 0.031 & 0.003 & 0.033 & 0.003 & 0.032 & 0.003 & 0.032 \\
\hline 0.5 & .081 & 0.137 & 0.079 & 0.136 & 0.079 & 0.13 & 0.080 & 0.139 & 0.080 & 0.138 & 0.079 & 0.134 \\
\hline 1.00 & 0.229 & 0.288 & 0.228 & 0.288 & 0.228 & 0.286 & 0.228 & 0.288 & 0.288 & 0.288 & 0.228 & 0.287 \\
\hline 1.50 & 0.295 & 0.355 & 0.293 & 0.354 & 0.292 & 0.35 & 0.295 & 0.353 & 0.294 & 0.352 & 0.291 & 0.351 \\
\hline 2.00 & 0.361 & 0.426 & 0.361 & 0.425 & 0.357 & 0.424 & 0.361 & 0.427 & 0.357 & 0.425 & 0.357 & 0.424 \\
\hline 2.50 & 0.430 & 0.478 & 0.429 & 0.478 & 0.427 & 0.477 & 0.433 & 0.481 & 0.430 & 0.479 & 0.426 & 0.478 \\
\hline \multicolumn{13}{|c|}{ SV2F: Two-factor continuous model } \\
\hline & \multicolumn{2}{|c|}{$z_{T P}$} & \multicolumn{2}{|c|}{$z_{T P, l m}$} & \multicolumn{2}{|c|}{$z_{T P, r m}$} & \multicolumn{2}{|c|}{$z_{Q P}$} & \multicolumn{2}{|c|}{$z_{\mathrm{QP}, l m}$} & \multicolumn{2}{|c|}{$z_{Q P, r m}$} \\
\hline Interval & 0.01 & 0.05 & 0.01 & 0.05 & 0.01 & 0.05 & 0.01 & 0.05 & 0.01 & 0.05 & 0.01 & 0.05 \\
\hline $30 \mathrm{~m}$ & 0.495 & 0.670 & 0.475 & 0.658 & 0.452 & 0.639 & 0.548 & 0.716 & 0.534 & 0.696 & 0.519 & 0.681 \\
\hline & 0.084 & 0.200 & 0.079 & 0.195 & 0.074 & 0.188 & 0.089 & 0.206 & 0.084 & 0.204 & 0.079 & 0.198 \\
\hline 3 minutes & 0.036 & 0.129 & 0.035 & 0.127 & 0.035 & 0.124 & 0.039 & 0.132 & 0.038 & 0.128 & 0.038 & 0.127 \\
\hline 1 minute & 0.045 & 0.124 & 0.044 & 0.123 & 0.044 & 0.119 & 0.045 & 0.125 & 0.044 & 0.124 & 0.043 & 0.122 \\
\hline
\end{tabular}

Fixed parameters: five-minute returns and $\lambda=0.014$ for SV1FJ model.

strategies to knock it out based on the behavior of $T \cdot B(M)$ for different $M$, but they all appear to us to entail additional assumptions and models that would be inconsistent with the nonparametric character of the jump detection strategies. So, for now, the daily statistics are perhaps more reliable than the full-sample statistics, at least in terms of their size, though the full-sample statistics offer some tantalizing longer term possibilities for development of consistent tests. For the related asymptotic results as both $T$ and $M$ go to infinity, see Corradi and 
Distaso (2004) in the framework of testing the specification of stochastic volatility models.

\section{Empirical Application}

The dataset consists of five-minute observations on the S\&P index; the cash data are from April 21, 1997, to October 22, 2002, and the futures data are from April 21, 1982 (the beginning of the S\&P futures contract), to December 9, 2002. We eliminated a few days where trading was thin and the market was open for a shortened session. Figure 11 shows plots of the daily price level and the daily geometric returns of the cash index and the index futures. For the within-day computations, we used five-minute data after applying a standard adjustment for the deterministic pattern of volatility over the trading day.

To investigate jumps, we first consider Figures 12 and 13, which show in each panel a time series plot of the five versions [Equations (5), (6), (7), (8), and (9)] of the $z_{T P, t}$ statistic computed over this dataset, along with the upper $0.99 \%$ and $0.999 \%$ critical values of the standard Gaussian distribution. From the Monte Carlo evidence generated in Section 4, the second to the last panels provide the more reliable evidence on days when large jumps occurred conditional on the
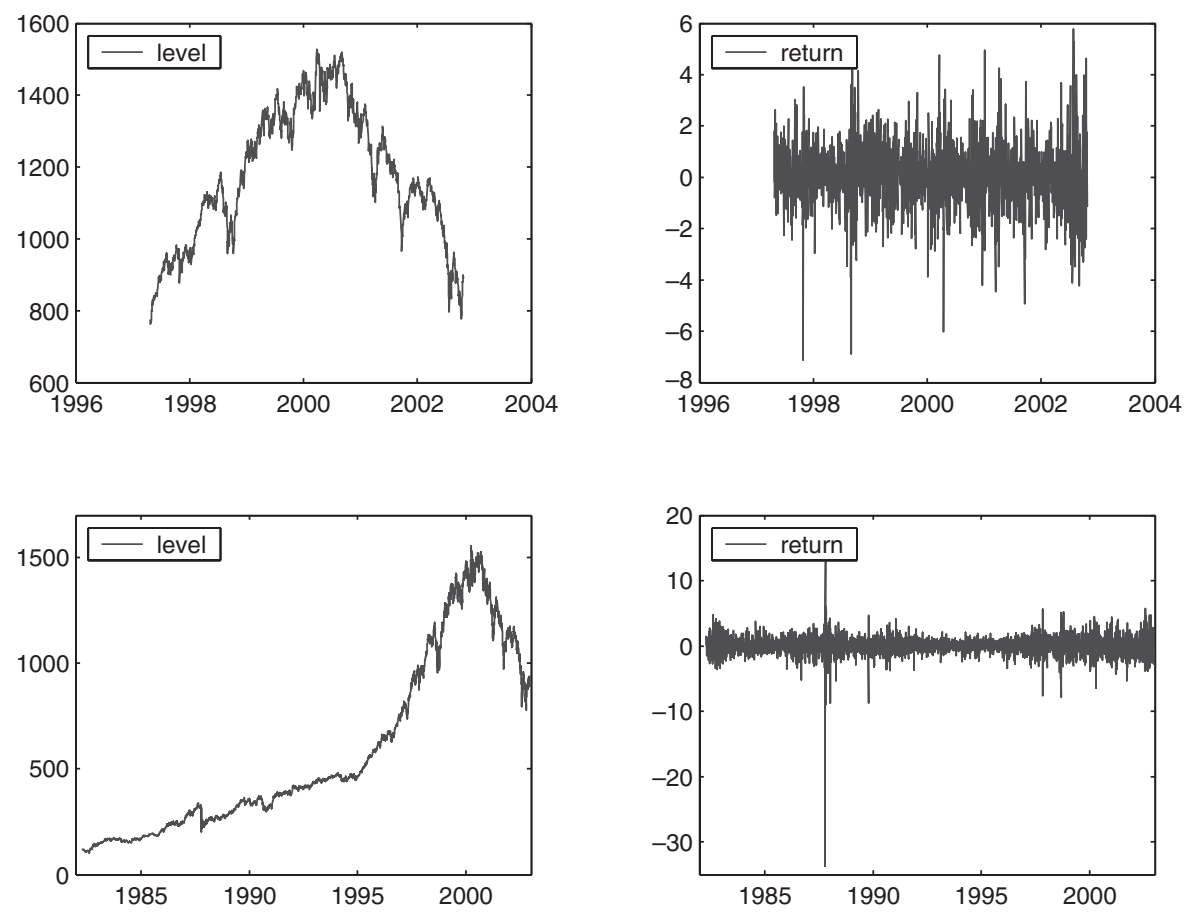

Figure 11 The top two panels show the daily closing price and the daily geometric return of the S\&P cash index, April 21, 1997-October 22, 2002. The bottom two panels show the daily closing price and the daily geometric return of the S\&P index futures, April 21, 1982-December 9, 2002. 

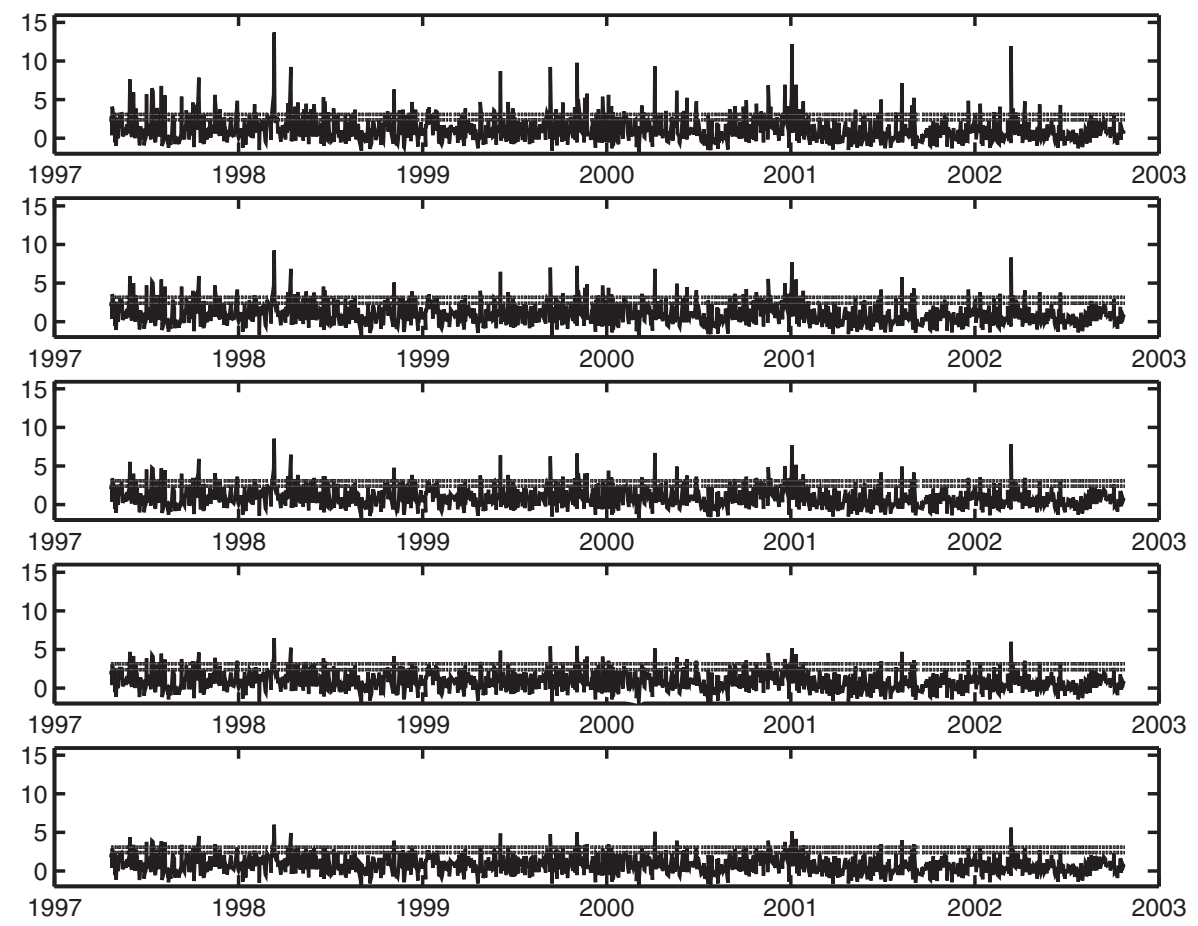

Figure 12 The five panels show observed values of the five daily jump statistics $-z_{T P, t}, z_{T P, l, t}$, $z_{T P, l m, t}, z_{T P, r, t}$ and $z_{T P, r m, t}$ - computed using the five-minute returns on the S\&P cash index, April 21, 1997-October 22, 2002. The horizontal lines are the upper 0.99 and 0.999 critical values of the standard Gaussian distribution.

SV1FJ model [Equation (23)]. Evidently the statistics indicate far more jumps than would be expected under a purely diffusive model satisfying the rather mild regularity conditions of Barndorff-Nielsen and Shephard (2006). Similarly there appear to be many more jumps than could possibly be generated as false jumps under the continuous SV2F model, which casts doubt on the validity of that model in describing the very high frequency character of stock prices.

We now consider the relative contribution of jumps to total price variance. Table 8 shows that the proportion of days that the daily z-statistics identify as having jumps is larger for the cash index than those for the index futures in the corresponding periods or in the full sample. Moreover, Table 9 shows that about $4.4 \%$ to $4.6 \%$ of the total realized variance comes from the jump component in the index futures, and, somewhat surprisingly, smaller than the value $(7.328 \%)$ in the cash index. A similar relationship holds for the full-sample statistics $\left(R J_{1: T}\right)$ as well. On the other hand, $R V_{1: T}$ and $B V_{1: T}$ for the index futures are larger than those for the cash index for both the shorter (1997-2002) and the longer samples (1982-2002). 


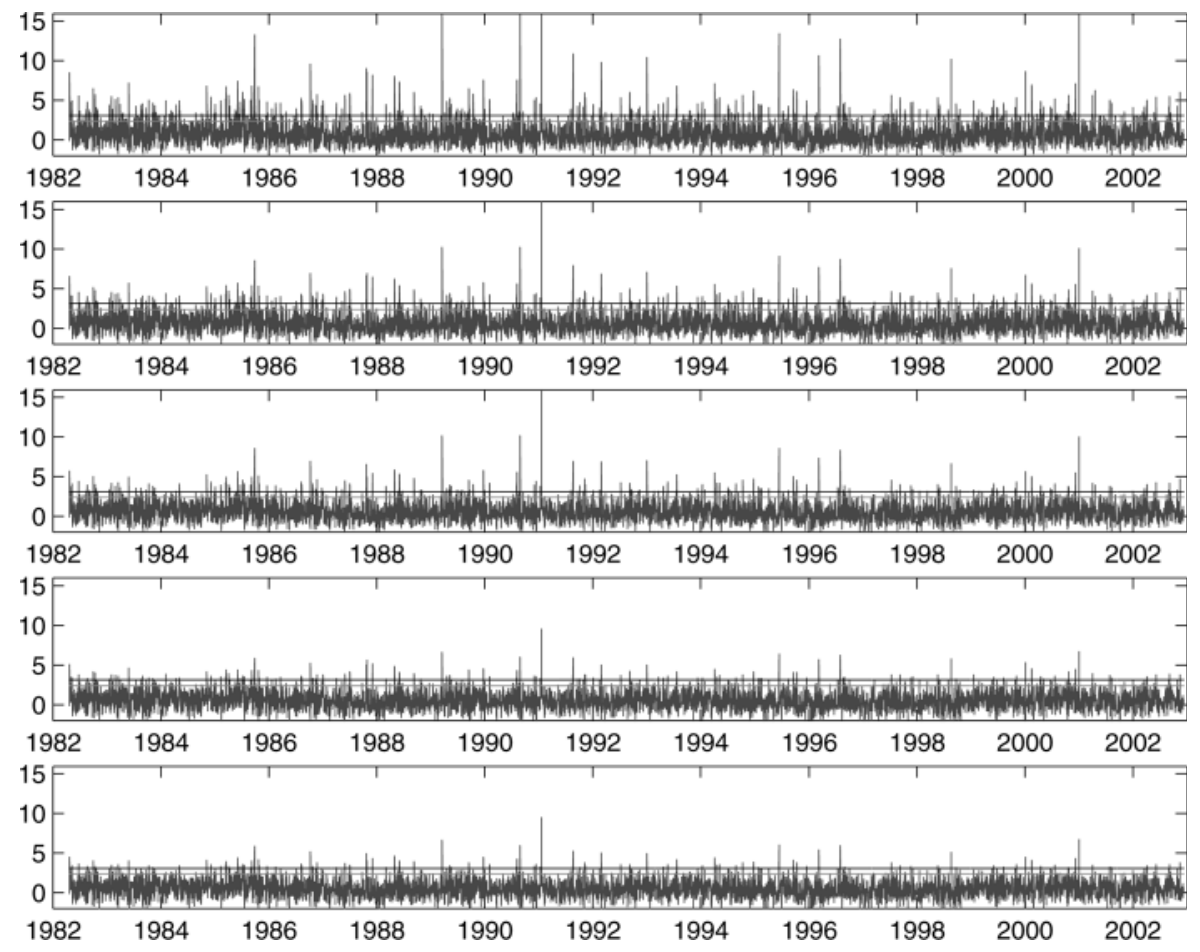

Figure 13 The five panels are the time series plots of the observed values of the daily statistics$z_{T P, t}, z_{T P, l, t}, z_{T P, l m, t}, z_{T P, r, t}$, and $z_{T P, r m, t}$-computed using the five-minute returns on the S\&P index futures, April 21, 1982-December 9, 2002. The horizontal lines are the upper 0.99 and 0.999 critical values of the standard Gaussian distribution.

Taken together, the results reported in Figures 12 and 13, along with those in Tables 8 and 9 , indicate that jumps are a statistically important component of aggregate stock price movements. This evidence is generated using statistical techniques validated in the Monte Carlo work of Section 4, which makes the case for jumps all that more compelling. The jumps appear to contribute about $4.4 \%$ to $7.3 \%$ to the total variance of daily stock price movements.

\section{Market Microstructure Noise}

Our empirical evidence on the jumps in the financial price process is consistent with the findings in Andersen, Bollerslev, and Diebold (2004). Eraker, Johannes, and Polson (2003) also find similar proportions of return variance due to jumps in S\&P 500 and NASDAQ 100 index returns using their jumps in volatility and returns models. These sets of findings suggest more instances of jumps and a higher jump contribution to total return variance than most of the existing literature. However, since we are using high-frequency data, the observed return 
Table 8 Proportion of days identified as jumps by the daily statistics.

\begin{tabular}{llllll}
\hline Level of & \multicolumn{5}{c}{} \\
significance & 0.500 & 0.950 & 0.990 & 0.995 & 0.999 \\
\hline \multicolumn{5}{c}{ S\&P 500 cash index $(04 / 21 / 1997-10 / 22 / 2002)$} \\
$z_{T P, t}$ & 0.74215 & 0.28488 & 0.18335 & 0.14609 & 0.10299 \\
$z_{T P, l, t}$ & 0.74215 & 0.26150 & 0.14536 & 0.12199 & 0.07159 \\
$z_{T P, l m, t}$ & 0.74215 & 0.24543 & 0.12929 & 0.09715 & 0.05698 \\
$z_{T P, r, t}$ & 0.74215 & 0.23886 & 0.11907 & 0.08836 & 0.04383 \\
$z_{T P, r m, t}$ & 0.74215 & 0.22060 & 0.09204 & 0.06866 & 0.03214 \\
& S\&P 500 index futures $(04 / 21 / 1997-10 / 22 / 2002)$ & \\
$z_{T P, t}$ & 0.66108 & 0.20918 & 0.11152 & 0.09402 & 0.05175 \\
$z_{T P, l, t}$ & 0.66108 & 0.18440 & 0.09329 & 0.06851 & 0.03717 \\
$z_{T P, l m, t}$ & 0.66108 & 0.17566 & 0.07726 & 0.05831 & 0.03061 \\
$z_{T P, r, t}$ & 0.66108 & 0.16618 & 0.06778 & 0.04227 & 0.02041 \\
$z_{T P, r m, t}$ & 0.66108 & 0.14942 & 0.05758 & 0.03499 & 0.01603 \\
& S\&P 500 index futures $(04 / 21 / 1982-12 / 09 / 2002)$ & \\
$z_{T P, t}$ & 0.64886 & 0.20960 & 0.11705 & 0.09583 & 0.05997 \\
$z_{T P, l, t}$ & 0.64886 & 0.18801 & 0.09526 & 0.07231 & 0.04319 \\
$z_{T P, l m, t}$ & 0.64886 & 0.17875 & 0.08427 & 0.06479 & 0.03779 \\
$z_{T P, r, t}$ & 0.64886 & 0.16815 & 0.07038 & 0.05110 & 0.02680 \\
$z_{T P, r m, t}$ & 0.64886 & 0.15523 & 0.06190 & 0.04223 & 0.02140 \\
\hline
\end{tabular}

series can be contaminated by market microstructure noise. That raises the issue of whether the jumps detected by the above statistics are true jumps or spurious ones induced by the market microstructure noise. To address this question, we conduct the following analysis of the effects of the market microstructure noise on jump detection.

\subsection{The Noise Structure}

We now assume the observed log price at time $t_{j}$ is

$$
p\left(t_{j}\right)=p^{*}\left(t_{j}\right)+u_{t, j},
$$

where $p^{*}(t)$ is the true continuous-time log price assumed to satisfy the conditions given in Section 1, and $u_{t, j}$ is the microstructure noise. We follow Aït-Sahalia, Mykland, and Zhang (2005), Zhang, Mykland, and Aït-Sahalia (2004), and Bandi and Russell (2005b), among others, and assume that the $u_{t, j}$ 's are i.i.d.; for tractability, we additionally assume the $u_{t, j}$ 's are $N\left(0, \sigma_{m n}^{2}\right)$. We discuss below the (important) deviations from this setup. For detailed studies on the realized volatility measures under non-i.i.d. market microstructure noise, see the above literature as well as Bandi and Russell (2005a), and Hansen and Lunde (2004a,b); a comprehensive survey is Barndorff-Nielsen and Shephard (2005b). 
Table 9 Summary of $R V, B V$, and $R J$.

\begin{tabular}{|c|c|c|c|c|}
\hline Statistics & $R V$ & $B V$ & $R V-B V$ & $R J$ \\
\hline \multicolumn{5}{|c|}{ S\&P 500 cash index $(04 / 21 / 1997-10 / 22 / 2002)$} \\
\hline Sample mean & 1.26702 & 1.18084 & 0.08618 & 0.07328 \\
\hline SD of mean & 0.04086 & 0.03746 & 0.00340 & 0.00294 \\
\hline Full-sample statistics & 1734.55618 & 1616.57673 & 117.97945 & 0.06802 \\
\hline \multicolumn{5}{|c|}{ S\&P 500 index futures $(04 / 21 / 1997-10 / 22 / 2002)$} \\
\hline Sample mean & 2.10718 & 2.01193 & 0.09525 & 0.04445 \\
\hline $\mathrm{SD}$ of mean & 0.06690 & 0.06266 & 0.00424 & 0.00279 \\
\hline Full-sample statistics & 2891.05330 & 2760.37224 & 130.68106 & 0.04520 \\
\hline \multicolumn{5}{|c|}{ S\&P 500 index futures $(04 / 21 / 1982-12 / 09 / 2002)$} \\
\hline Sample mean & 1.63393 & 1.46074 & 0.17319 & 0.04562 \\
\hline SD of mean & 0.21083 & 0.12375 & 0.08708 & 0.00152 \\
\hline Full-sample statistics & 8473.55466 & 7575.38272 & 898.17194 & 0.10600 \\
\hline
\end{tabular}

Under these assumptions, the observed return is

$$
r_{t, j}=r_{t, j}^{*}+\varepsilon_{t, j}
$$

where $r_{t, j}^{*}$ is the true geometric return based on $p^{*}\left(t_{j}\right)$ and $\varepsilon_{t, j}$ is an MA(1) process with $\operatorname{var}\left(\varepsilon_{t, j}\right)=2 \sigma_{m n}^{2}$ and $\operatorname{cov}\left(\varepsilon_{t, j}, \varepsilon_{t, j-1}\right)=-\sigma_{m n}^{2}$. As above, we assume equally spaced sampling, where $\delta=t_{j}-t_{j-1}$ denotes the sampling interval and is equal to $\frac{1}{M}$. Let $\sigma_{d}^{2}$ denote the unconditional daily variance of the true returns, so, as noted by Aït-Sahalia, Mykland, and Zhang (2005),

$$
\pi_{m n}(\delta)=\frac{2 \sigma_{m n}^{2}}{\sigma_{d}^{2} \delta+2 \sigma_{m n}^{2}}
$$

is the proportion of the variance of the return over the interval $\left[t_{j}-\delta, t_{j}\right]$ attributable to the microstructure noise.

\subsection{Staggered Returns}

Recall from Section 1 the definition of bipower variation,

$$
B V_{t}=\mu_{1}^{-2}\left(\frac{M}{M-1}\right) \sum_{j=2}^{M}\left|r_{t, j-1}\right|\left|r_{t, j}\right| .
$$

The effect of the microstructure noise introduced in Subsection 6.1 is to induce correlation in the two adjacent returns, $r_{t, j-1}$ and $r_{t, j}$, appearing in this summation. Anderson, Bollerslev, and Diebold (2004) suggest breaking the correlation by using staggered returns as in $\left|r_{t, j-2}\right|\left|r_{t, j}\right|$, or more generally as in $\left|r_{t, j-(1+i)}\right|\left|r_{t, j}\right|$, where the nonnegative integer $i$ denotes the offset. The asymptotics of various bipower measures using staggered returns are studied in depth by 
Barndorff-Nielsen and Shephard (2004a). Here we consider the generalized bipower measure based on staggered returns,

$$
B V_{i, t}=\mu_{1}^{-2}\left(\frac{M}{M-1-i}\right) \sum_{j=2+i}^{M}\left|r_{t, j-(1+j)}\right|\left|r_{t, j}\right|, \quad i \geq 0 .
$$

The above reduces to the bipower variation defined in Section 1 if $i=0$. Likewise, we consider the generalized tri-power quarticity measure,

$$
\operatorname{TP}_{i, t}=M \mu_{4 / 3}^{-3}\left(\frac{M}{M-2(1+i)}\right) \sum_{j=1+2(1+i)}^{M}\left|r_{t, j-2(1+i)}\right|^{4 / 3}\left|r_{t, j-(1+i)}\right|^{4 / 3}\left|r_{t, j}\right|^{4 / 3}, \quad i \geq 0,
$$

and the quad-power quarticity measure,

$$
Q P_{i, t}=M \mu_{1}^{-4}\left(\frac{M}{M-3(1+i)}\right) \sum_{j=1+3(1+i)}^{M}\left|r_{t, j-3(1+i)}\right|\left|r_{t, j-2(1+i)}\right|\left|r_{t, j-(1+i)}\right|\left|r_{t, j}\right|, \quad i \geq 0 \text {. }
$$

With $B V_{i, t}, T P_{i, t}, Q P_{i, t}$ used in place of $B V_{t}, T P_{t}, Q P_{t}$, the asymptotic theory of Section 2 for the various $z$-test statistics of Section 3 is exactly the same for each fixed $i$ and $M \rightarrow \infty$.

\subsection{Some Analytics}

To analyze the relationship between the realized variance and the bipower variation in the presence of microstructure noise, we retain the simple standard Gaussian i.i.d. structure of Subsection 6.1 above; later we discuss the implications of deviations from this setup. With these assumptions for the noise, assuming no leverage, working conditional on the volatility process, and with the $t$ subscript suppressed, we can write the $j$ th within-day return as

$$
r_{j} \stackrel{D}{=}\left(\sigma_{j}^{2} \delta+\sigma_{m n}^{2}\right)^{\frac{1}{2}} Z_{j},
$$

where the $Z_{j}^{\prime}$ s are standard Gaussian random variables and $\sigma_{j}^{2}$ is the integrated variance averaged over the sampling interval of width $\delta$. The correlations of the $Z_{j}^{\prime}$ s are

$$
\rho_{j, k} \equiv \operatorname{corr}\left(Z_{j},-Z_{j-k}\right)=\left\{\begin{array}{r}
\frac{-\sigma_{m n}^{2}}{\sqrt{\left(\sigma_{j}^{2} \delta+\sigma_{m n}^{2}\right)\left(\sigma_{j-k}^{2} \delta+\sigma_{m n}^{2}\right)}}|k|=1, \\
0|k| \geq 1 .
\end{array}\right.
$$

All of the jump test $z$-statistics displayed in Section 3 have the common structure of a studentized measure of discrepancy

$$
z=\frac{D(R V, B V)}{\sqrt{\operatorname{Avar}[D(R V, B V)]}},
$$


where the numerator of the $z$-statistic, $D(\cdot, \cdot)$, is a measure of the discrepancy between the realized variance $R V$ and the bipower variation $B V$, and the denominator is the square root of the asymptotic variance of the discrepancy under the Barndorff-Nielsen and Shephard theory. The asymptotic variance of $D(\cdot, \cdot)$ depends on the integrated quarticity, which is estimated by either the realized tri-power quarticity or the realized quad-power quarticity.

To gain insight into the behavior of $z$-statistic [Equation (34)], we consider the conditional expectations, given the volatility process, of the numerator and the realized tri-power quarticity term for the denominator in Equation (34) for the basic case where the discrepancy $D(\cdot, \cdot)$ is simply the difference, as in Equation (5). For simplicity, we ignore the small sample adjustment $M /(M-1)$ along with end effects due to lagging, and we presume the same number of terms $M=1 / \delta$ in the summations.

The relevant conditional expectation for the numerator is thus

$$
\mathrm{E}_{\sigma}\left(\sum_{1}^{M} r_{j}^{2}\right)-\mathrm{E}_{\sigma}\left(\frac{\pi}{2} \sum_{1}^{M}\left|r_{j-(1+i)}\right|\left|r_{j}\right|\right),
$$

where, as before, $i \geq 0$ is the offset, and $\mathrm{E}_{\sigma}$ denotes the expectation computed conditional on the trajectory of volatility. From Equation (32) we have that Equation (35) is

$$
\begin{aligned}
& \sum_{1}^{M}\left(\sigma_{j}^{2} \delta+\sigma_{m n}^{2}\right)^{\frac{1}{2}}\left[\left(\sigma_{j}^{2} \delta+\sigma_{m n}^{2}\right)^{\frac{1}{2}}-\left(\sigma_{j-(1+i)}^{2} \delta+\sigma_{m n}^{2}\right)^{\frac{1}{2}}\right] \\
& +\sum_{1}^{M}\left(\sigma_{j}^{2} \delta+\sigma_{m n}^{2}\right)^{\frac{1}{2}}\left(\sigma_{j-(1+i)}^{2} \delta+\sigma_{m n}^{2}\right)^{\frac{1}{2}}\left[\frac{2}{\pi}-g\left(\rho_{j, 1+i}\right)\right] \frac{\pi}{2},
\end{aligned}
$$

where

$$
g(\rho)=\mathrm{E}\left(\left|\left(1-\rho^{2}\right)^{\frac{1}{2}} Z_{a}+\rho Z_{b}\right|\left|Z_{b}\right|\right), \quad Z_{a}, Z_{b}, \text { independent } \mathrm{N}(0,1),
$$

is the expected value of the product of the absolute values of two correlated standard Guassian random variables. The function $g(\rho)$ is symmetric about zero with $g(0)=2 / \pi, g(1)=1$, and it is easily numerically evaluated. For an explicit expression for $g(\rho)$ and its properties, see Barndorff-Nielsen and Shephard (2004a) Theorem 3 and Remark 5. Application of the mean value theorem to the square-root function $(\cdot)^{\frac{1}{2}}$ appearing in the first square bracket in Equation (36) gives

$$
\begin{aligned}
\mathrm{E}_{\sigma} & \left(\sum_{1}^{M} r_{j}^{2}\right)-\mathrm{E}_{\sigma}\left(\frac{\pi}{2} \sum_{1}^{M}\left|r_{j-(1+i)}\right|\left|r_{j}\right|\right) \\
& =\sum_{1}^{M} \frac{1}{2}\left[\frac{\sigma_{j}^{2} \delta+\sigma_{m n}^{2}}{\sigma_{j}^{2} \delta+\sigma_{m n}^{2}+\phi_{j}\left(\sigma_{j}^{2}-\sigma_{j-(1+i)}^{2}\right)^{\delta}}\right]^{\frac{1}{2}}\left(\sigma_{j}^{2}-\sigma_{j-(1+i)}^{2}\right) \delta \\
& +\sum_{1}^{M}\left(\sigma_{j}^{2} \delta+\sigma_{m n}^{2}\right)^{\frac{1}{2}}\left(\sigma_{j-(1+i)}^{2} \delta+\sigma_{m n}^{2}\right)^{\frac{1}{2}}\left[\frac{2}{\pi}-g\left(\rho_{j, 1+i}\right)\right] \frac{\pi}{2},
\end{aligned}
$$


where $\left|\phi_{j}\right|<1$. Hence the conditional expectation of the numerator of the $z$-statistic [Equation (34)] is the sum of two terms,

$$
\mathrm{E}_{\sigma}\left(\sum_{1}^{M} r_{j}^{2}\right)-\mathrm{E}_{\sigma}\left(\frac{\pi}{2} \sum_{1}^{M}\left|r_{j-(1+i)}\right|\left|r_{j}\right|\right)=A_{1}+A_{2}
$$

where

$$
\begin{aligned}
& A_{1}=\frac{1}{M} \sum_{1}^{M} \frac{1}{2}\left[\frac{\sigma_{j}^{2} \delta+\sigma_{m n}^{2}}{\sigma_{j}^{2} \delta+\sigma_{m n}^{2}+\phi_{j}\left(\sigma_{j}^{2}-\sigma_{j-(1+i)}^{2}\right)^{\delta}}\right]^{\frac{1}{2}}\left(\sigma_{j}^{2}-\sigma_{j-(1+i)}^{2}\right), \\
& A_{2}=\sum_{1}^{M}\left(\sigma_{j}^{2} \delta+\sigma_{m n}^{2}\right)^{\frac{1}{2}}\left(\sigma_{j-(1+i)}^{2} \delta+\sigma_{m n}^{2}\right)^{\frac{1}{2}}\left[\frac{2}{\pi}-g\left(\rho_{j, 1+i}\right)\right] \frac{\pi}{2},
\end{aligned}
$$

and recall $\delta=\frac{1}{M}$. For any fixed $i \geq 0$, the first term $A_{1}$ converges to zero as $\delta$ goes to zero, so it is robust with respect to the microstructure noise. As for the second term, if returns are not staggered so $i=0$, it does not converge to zero, and the numerator is biased in the negative direction against finding jumps. In contrast, if returns are staggered, $i>0$, then, because of the i.i.d. assumption, $\rho_{j, 1+i}=0$ and the term $A_{2}$ vanishes, leaving only the term $A_{1}$. It is important to keep in mind that the Gaussian assumption determines the characteristics of the function $g(\rho)$ in Equation (37), and so that assumption plays a key role in our results regarding the behavior of the term $A_{2}$.

We now consider the denominator of Equation (34), and we examine the case where the realized tri-power quarticity is used to estimate the asymptotic variance in Equation (34). The relevant conditional expectation is

$$
\mu_{4 / 3}^{-3} \mathrm{E}_{\sigma}\left(\sum_{j=1}^{M}\left|r_{j-2(1+i)}\right|^{4 / 3}\left|r_{j-(1+i)}\right|^{4 / 3}\left|r_{j}\right|^{4 / 3}\right) .
$$

Using the representation of Equation (32), the above becomes

$$
\sum_{j=1}^{M}\left(\sigma_{j-2(1+i)}^{2} \delta+\sigma_{m n}^{2}\right)^{2 / 3}\left(\sigma_{j-(1+i)}^{2} \delta+\sigma_{m n}^{2}\right)^{2 / 3}\left(\sigma_{j}^{2} \delta+\sigma_{m n}^{2}\right)^{2 / 3} \mu_{4 / 3}^{-3} h\left(\rho_{j-(1+i), 1+i}, \rho_{j, 1+i}\right)
$$

where $\rho_{j, k}$ is defined in Equation (33), and the function $h$ is given by

$$
h\left(\rho_{a}, \rho_{b}\right)=\mathrm{E}\left(\left|\left(1-\frac{\rho_{a}^{2}}{1-\rho_{b}^{2}}\right)^{\frac{1}{2}} Z_{a}+\frac{\rho_{a}}{\sqrt{1-\rho_{b}^{2}}} Z_{b}\right|^{4 / 3} \cdot\left|\left(1-\rho_{b}^{2}\right) \frac{1}{2} Z_{b}+\rho_{b} Z_{c}\right|^{4 / 3} \cdot\left|Z_{c}\right|^{4 / 3}\right) .
$$

The function $h$ is equivalently written as 


$$
h\left(\rho_{a}, \rho_{b}\right)=E\left(\left|Y_{a}\right|^{4 / 3}\left|Y_{b}\right|^{4 / 3}\left|Y_{c}\right|^{4 / 3}\right),
$$

where $Y_{a}, Y_{b}, Y_{c}$ are three standard Gaussian random variables with pairwise correlations $\operatorname{corr}\left(Y_{a}, Y_{b}\right)=\rho_{a}, \operatorname{corr}\left(Y_{b}, Y_{c}\right)=\rho_{b}, \operatorname{corr}\left(Y_{a}, Y_{c}\right)=0$. The function is easily numerically evaluated.

Equation (42) indicates that the effect of the microstructure noise is to inflate the realized tri-power quarticity for two reasons, although the inflation can be substantially mitigated by staggering returns. First, the noise variance $\sigma_{m n}^{2}$ inflates terms like $\left(\sigma_{j}^{2} \delta+\sigma_{m n}^{2}\right)^{\frac{1}{2}}$. Second, if there is no staggering, then adjacent returns are negatively correlated, so their magnitudes are positively correlated in Equation (41), and therefore

$$
\mu_{4 / 3}^{-3} h\left(\rho_{j-1,1}, \rho_{j, 1}\right)>1 .
$$

Table 10 shows the value of the factor $\mu_{4 / 3}^{-3} h$ for different values of the correlations, and it suggests the inflation of the realized tri-power quarticity can be substantial, as high as $25 \%$ to $39 \%$. On the other hand, if the returns are staggered via $i \geq 1$, then the inflation due to the correlation of adjacent returns is absent.

The upshot of our analytical analysis is that without staggering, our results suggest the jump test statistics to be biased downward, that is, in favor of finding fewer jumps in the presence of market microstructure noise. The reason is that the numerator of the test statistic is negatively biased. At the same time, the noise inflates the estimate of the integrated quarticity used to form the estimate of scale in the denominator of Equation (34). The two effects together bias the $z_{T P}$ statistic against rejection. We expect the same logic to work for the other forms of the $z$-statistics.

If returns are staggered, $i \geq 1$, in forming the requisite power measures of Equations (29)-(31), then the term $A_{2}$ is knocked out of the numerator, and $\mu_{4 / 3}^{-3} h=1$. In this case, the asymptotic approximation of the Barndorff-Nielsen and Shephard theory is expected to be more accurate. Nonetheless, one must keep in mind the presumption of i.i.d. Gaussian microstructure noise. If the micro-

Table 10 Values of $\mu_{4 / 3}^{-3} h\left(\rho_{a}, \rho_{b}\right)$.

\begin{tabular}{rcccccc}
\hline & \multicolumn{7}{c}{$\rho_{b}$} \\
\cline { 2 - 7 }$\rho_{\alpha}$ & -0.50 & -0.40 & -0.30 & -0.20 & -0.10 & 0.00 \\
\hline-0.50 & 1.39 & 1.32 & 1.27 & 1.23 & 1.21 & 1.21 \\
-0.40 & 1.32 & 1.25 & 1.19 & 1.15 & 1.13 & 1.13 \\
-0.30 & 1.27 & 1.19 & 1.13 & 1.08 & 1.07 & 1.06 \\
-0.20 & 1.23 & 1.15 & 1.08 & 1.04 & 1.02 & 1.02 \\
-0.10 & 1.21 & 1.13 & 1.07 & 1.02 & 1.01 & 1.00 \\
0.00 & 1.21 & 1.13 & 1.06 & 1.02 & 1.00 & 1.00 \\
\hline
\end{tabular}

This table is computed by numerical integration using Gauss-Hermite quadrature. 
structure noise is non-Gaussian, then the decomposition of Equations (39) and (40) is unavailable, although the results are still suggestive; we defer non-Gaussian noise to future work. If the microstructure noise is Gaussian, but serially correlated, such as an $\mathrm{MA}(k)$ process, then one can reasonably expect an appropriately long lag $i$ for the staggering to work, but again we defer formal verification to later work.

Clearly our separate analysis of the conditional expectations of the numerator and denominator of the $z$ jump statistic can only provide some guidance of what to expect for the $z$-statistic in the presence of microstructure noise. A formal theoretical analysis of the $z$-statistic itself would entail complicated higher order approximations, so we turn to a Monte Carlo assessment instead.

\subsection{Monte Carlo Assessment of the Effects of Microstructure Noise}

We need to run the simulations over a plausibly wide range of values for the standard deviation $\sigma_{m n}$ of the microstructure noise that appears in Equation (26). To calibrate $\sigma_{m n}$, we use Equation (28) with a presumed return volatility of $1 \%$ per day, so that

$$
\pi_{m n}(5 / 390)=\frac{2 \sigma_{m n}^{2}}{1 \cdot(5 / 390)+2 \sigma_{m n}^{2}}
$$

is the proportion of the variance of the five-minute return attributable to microstructure noise for a market open 6.50 hours per day. We consider values of $\pi_{m n}(5 / 390)$ ranging from 0.00 to 0.50 in the increment of 0.10 , which thereby determines the grid of values of $\sigma_{m n}$ and also values of $\pi_{m n}(\delta)$ for all other sampling frequencies as well; see Table 11. The upper value of $\pi_{m n}(5 / 390)=$ 0.50 implies that $50 \%$ of the variance of the five-minute return is accounted for by the noise, which appears to be more than adequate for an index cash or an index future like those usually found in most empirical work [e.g., Kaul and Nimalendran (1990), Conrad, Kaul and Nimalendran (1991), Hasbrouck (1993) and Madhavan, Richardson, and Roomans (1997), among others].

The following subsections summarize the Monte Carlo findings with the market microstructure noise.

6.4.1 Size. Table 12 shows the size of the three $z_{t p}$ statistics under different noise sizes $\left(\sigma_{m n}\right)$ and sampling intervals, with $i=0,1$, and 2 . It is clear from this table

Table 11 Proportions of noise contribution under different sampling intervals in Monte Carlo.

\begin{tabular}{lllllll}
\hline Interval & \multicolumn{7}{c}{$\pi_{m n}$} \\
\hline 1 minute & 0.000 & 0.357 & 0.556 & 0.682 & 0.769 & 0.833 \\
3 minutes & 0.000 & 0.156 & 0.294 & 0.417 & 0.526 & 0.625 \\
5 minutes & 0.000 & 0.100 & 0.200 & 0.300 & 0.400 & 0.500 \\
30 minutes & 0.000 & 0.018 & 0.040 & 0.067 & 0.100 & 0.143 \\
$\sigma_{\mathrm{mn}}$ & 0.000 & 0.027 & 0.040 & 0.052 & 0.065 & 0.080 \\
\hline
\end{tabular}


Table 12 Size of different jump statistics under market microstructure noise.

\begin{tabular}{|c|c|c|c|c|c|c|c|}
\hline \multirow{2}{*}{ Interval } & & \multicolumn{6}{|c|}{$\sigma_{m n}$} \\
\hline & & 0.000 & 0.027 & 0.040 & 0.052 & 0.065 & 0.080 \\
\hline \multirow{4}{*}{1 minute } & & & $(i=0)$ & & & & \\
\hline & $z_{T P, t}$ & 0.020 & 0.007 & 0.002 & 0.001 & 0.000 & 0.000 \\
\hline & $z_{T P, l m, t}$ & 0.014 & 0.005 & 0.002 & 0.000 & 0.000 & 0.000 \\
\hline & $z_{T P, r m, t}$ & 0.012 & 0.004 & 0.001 & 0.000 & 0.000 & 0.000 \\
\hline \multirow[t]{2}{*}{3 minutes } & $z_{T P, t}$ & 0.032 & 0.027 & 0.020 & 0.014 & 0.009 & 0.005 \\
\hline & $z_{T P, l m, t}$ & 0.019 & 0.017 & 0.013 & 0.008 & 0.005 & 0.003 \\
\hline \multirow{3}{*}{5 minutes } & $z_{T P, r m, t}$ & 0.012 & 0.011 & 0.008 & 0.005 & 0.003 & 0.002 \\
\hline & $z_{T P, t}$ & 0.041 & 0.038 & 0.034 & 0.029 & 0.022 & 0.017 \\
\hline & $z_{T P, l m, t}$ & 0.023 & 0.022 & 0.019 & 0.015 & 0.012 & 0.009 \\
\hline \multirow{4}{*}{30 minutes } & $z_{T P, r m, t}$ & 0.014 & 0.014 & 0.011 & 0.009 & 0.007 & 0.005 \\
\hline & $z_{T P, t}$ & 0.107 & 0.107 & 0.107 & 0.107 & 0.107 & 0.107 \\
\hline & $z_{T P, l m, t}$ & 0.047 & 0.047 & 0.047 & 0.046 & 0.046 & 0.046 \\
\hline & $z_{T P, r m, t}$ & 0.014 & 0.015 & 0.015 & 0.015 & 0.014 & 0.014 \\
\hline \multirow{4}{*}{1 minute } & & & $(i=1)$ & & & & \\
\hline & $z_{T P, t}$ & 0.021 & 0.020 & 0.021 & 0.021 & 0.022 & 0.022 \\
\hline & $z_{T P, l m, t}$ & 0.015 & 0.015 & 0.015 & 0.015 & 0.015 & 0.015 \\
\hline & $z_{T P, r m, t}$ & 0.012 & 0.012 & 0.011 & 0.012 & 0.012 & 0.012 \\
\hline \multirow[t]{2}{*}{3 minutes } & $z_{T P, t}$ & 0.031 & 0.034 & 0.033 & 0.032 & 0.032 & 0.032 \\
\hline & $z_{T P, l m, t}$ & 0.020 & 0.020 & 0.021 & 0.020 & 0.019 & 0.019 \\
\hline \multirow{3}{*}{5 minutes } & $z_{T P, r m, t}$ & 0.014 & 0.014 & 0.014 & 0.013 & 0.013 & 0.013 \\
\hline & $z_{T P, t}$ & 0.042 & 0.041 & 0.041 & 0.042 & 0.041 & 0.041 \\
\hline & $z_{T P, l m, t}$ & 0.024 & 0.023 & 0.024 & 0.024 & 0.024 & 0.022 \\
\hline \multirow{4}{*}{30 minutes } & $z_{T P, r m, t}$ & 0.014 & 0.014 & 0.015 & 0.014 & 0.014 & 0.014 \\
\hline & $z_{T P, t}$ & 0.129 & 0.128 & 0.128 & 0.127 & 0.127 & 0.126 \\
\hline & $z_{T P, l m, t}$ & 0.058 & 0.057 & 0.058 & 0.059 & 0.058 & 0.057 \\
\hline & $z_{T P, r m, t}$ & 0.021 & 0.020 & 0.020 & 0.020 & 0.020 & 0.020 \\
\hline \multirow{4}{*}{1 minutes } & & & $(i=2$ & & & & \\
\hline & $z_{T P, t}$ & 0.022 & 0.021 & 0.021 & 0.022 & 0.023 & 0.023 \\
\hline & $z_{T P, l m, t}$ & 0.016 & 0.015 & 0.015 & 0.016 & 0.016 & 0.017 \\
\hline & $z_{T P, r m, t}$ & 0.013 & 0.011 & 0.011 & 0.012 & 0.013 & 0.013 \\
\hline \multirow[t]{2}{*}{3 minutes } & $z_{T P, t}$ & 0.034 & 0.035 & 0.034 & 0.035 & 0.035 & 0.035 \\
\hline & $z_{T P, l m, t}$ & 0.022 & 0.021 & 0.021 & 0.022 & 0.021 & 0.022 \\
\hline \multirow{3}{*}{5 minutes } & $z_{T P, r m, t}$ & 0.015 & 0.014 & 0.014 & 0.015 & 0.015 & 0.015 \\
\hline & $z_{T P, t}$ & 0.044 & 0.042 & 0.043 & 0.043 & 0.043 & 0.042 \\
\hline & $z_{T P, l m, t}$ & 0.025 & 0.024 & 0.024 & 0.024 & 0.024 & 0.024 \\
\hline \multirow{4}{*}{30 minutes } & $z_{T P, r m, t}$ & 0.016 & 0.014 & 0.014 & 0.015 & 0.015 & 0.015 \\
\hline & $z_{T P, t}$ & 0.144 & 0.145 & 0.145 & 0.146 & 0.146 & 0.145 \\
\hline & $z_{T P, l m, t}$ & 0.070 & 0.069 & 0.069 & 0.068 & 0.068 & 0.068 \\
\hline & $z_{T P, r m, t}$ & 0.025 & 0.026 & 0.026 & 0.025 & 0.025 & 0.026 \\
\hline
\end{tabular}

Fixed parameters: level of significance $=0.010, \alpha_{\nu}=-0.100$. 
that without staggering, the z-statistics' rejection frequency is seriously downward biased at the 1-minute level, but not at the 30-minute level, since the market microstructure noise impact decreases as the sampling interval increases. Moreover, the larger the noise size, the more serious the underrejection is, as explained in Section 6.3.

Staggering in $B V_{t}$ and $T P_{t}$, one the other hand, restores the nominal size and makes the rejection rate robust to the noise proportion. Extra lagging $(i=2)$ retains the robustness feature, but leads to a little overrejection. Thus, to achieve the correct empirical size of the statistics, we can only stagger the returns up to the level that breaks the serial dependence of the observed returns induced by the market microstructure noise. Any extra lagging might introduce finite-sample bias due to the longer interval covered in each return product term, making the asymptotic approximation worse.

6.4.2 Power and Jump Detection. Table 13 summarizes the power of the three $z_{T P}$ statistics and Table 14 shows the jump detection rate of the $z_{T P, r m, t}$ statistic under different noise sizes and sampling intervals, with $i=0,1$, and 2 . Without staggering, noise size reduces the power and the jump detection rate of the statistics due to the noise contribution of the total price variance, which has different characteristics from those of the jump part. Staggering helps to improve the jump detection rate at high frequency levels (one-minute and three-minute returns).

We did the same experiment for the constant volatility setup as the benchmark case. It turns out that the above features hold true under the constant volatility setup, showing the robustness of the results.

\subsection{Empirical Data Revisited}

The Monte Carlo evidence points toward the conclusion in Section 5 that the jumps detected in the S\&P data are bona fide jumps instead of market microstructure noise. We reconduct the same calculation, using staggered returns from $i=0$ to $i=$ 2. Here we only use the S\&P 500 index futures, because the cash index is made up of 500 underlying stocks without a sensible notion of the market microstructure noise. For comparability, we use the same subperiods as those in Section 5.

The empirical evidence, especially over the entire sample period April 21, 1982, to December 9, 2002, is generally consistent with the above analytical and Monte Carlo findings. Staggering suggests a slightly more important role for jumps both in terms of the number of jump days and the jump contribution in the total price variance, as exhibited in Tables 15 and 16.

\section{CONCLUSION}

The Monte Carlo evidence suggests that, under the arguably realistic scenarios considered here, the recently developed $z$-tests for jumps perform impressively and are not easily fooled. Computed on a daily basis in ratio form, with a 
Table 13 Rejection frequencies of different jump statistics under market microstructure noise.

\begin{tabular}{|c|c|c|c|c|c|c|c|}
\hline \multirow{2}{*}{ Interval } & & \multicolumn{6}{|c|}{$\sigma_{m n}$} \\
\hline & & 0.000 & 0.027 & 0.040 & 0.052 & 0.065 & 0.080 \\
\hline \multirow{4}{*}{1 minute } & & & $(i=$ & & & & \\
\hline & $z_{T P, t}$ & 0.032 & 0.018 & 0.012 & 0.010 & 0.008 & 0.007 \\
\hline & $z_{T P, l m, t}$ & 0.026 & 0.016 & 0.011 & 0.009 & 0.008 & 0.007 \\
\hline & $z_{T P, r m, t}$ & 0.023 & 0.015 & 0.011 & 0.009 & 0.008 & 0.006 \\
\hline \multirow[t]{3}{*}{3 minutes } & $z_{T P, t}$ & 0.042 & 0.036 & 0.030 & 0.023 & 0.017 & 0.013 \\
\hline & $z_{T P, l m, t}$ & 0.029 & 0.027 & 0.022 & 0.017 & 0.013 & 0.010 \\
\hline & $z_{T P, r m, t}$ & 0.022 & 0.021 & 0.018 & 0.014 & 0.011 & 0.009 \\
\hline \multirow[t]{3}{*}{5 minutes } & $z_{T P, t}$ & 0.050 & 0.048 & 0.043 & 0.037 & 0.030 & 0.025 \\
\hline & $z_{T P, l m, t}$ & 0.033 & 0.032 & 0.028 & 0.024 & 0.020 & 0.017 \\
\hline & $z_{T P, r m, t}$ & 0.023 & 0.023 & 0.020 & 0.018 & 0.015 & 0.013 \\
\hline \multirow[t]{4}{*}{30 minutes } & $z_{T P, t}$ & 0.112 & 0.113 & 0.113 & 0.112 & 0.112 & 0.111 \\
\hline & $z_{T P, l m, t}$ & 0.052 & 0.052 & 0.052 & 0.051 & 0.050 & 0.050 \\
\hline & $z_{T P, r m, t}$ & 0.018 & 0.019 & 0.019 & 0.018 & 0.018 & 0.018 \\
\hline & & & $(i=1$ & & & & \\
\hline \multirow[t]{3}{*}{1 minute } & $z_{T P, t}$ & 0.032 & 0.031 & 0.031 & 0.031 & 0.031 & 0.030 \\
\hline & $z_{T P, l m, t}$ & 0.026 & 0.026 & 0.025 & 0.025 & 0.025 & 0.024 \\
\hline & $z_{T P, r m, t}$ & 0.023 & 0.022 & 0.022 & 0.021 & 0.021 & 0.020 \\
\hline \multirow[t]{3}{*}{3 minutes } & $z_{T P, t}$ & 0.042 & 0.044 & 0.043 & 0.042 & 0.041 & 0.040 \\
\hline & $z_{T P, l m, t}$ & 0.030 & 0.031 & 0.030 & 0.029 & 0.028 & 0.027 \\
\hline & $z_{T P, r m, t}$ & 0.024 & 0.024 & 0.024 & 0.023 & 0.022 & 0.021 \\
\hline \multirow[t]{3}{*}{5 minutes } & $z_{T P, t}$ & 0.052 & 0.051 & 0.051 & 0.051 & 0.049 & 0.049 \\
\hline & $z_{T P, l m, t}$ & 0.033 & 0.033 & 0.033 & 0.033 & 0.032 & 0.030 \\
\hline & $z_{T P, r m, t}$ & 0.024 & 0.023 & 0.024 & 0.022 & 0.022 & 0.021 \\
\hline \multirow[t]{4}{*}{30 minutes } & $z_{T P, t}$ & 0.134 & 0.133 & 0.133 & 0.132 & 0.132 & 0.131 \\
\hline & $z_{T P, l m, t}$ & 0.063 & 0.062 & 0.063 & 0.063 & 0.062 & 0.061 \\
\hline & $z_{T P, r m, t}$ & 0.024 & 0.024 & 0.024 & 0.024 & 0.023 & 0.023 \\
\hline & & & $(i=2$ & & & & \\
\hline \multirow[t]{3}{*}{1 minute } & $z_{T P, t}$ & 0.034 & 0.032 & 0.031 & 0.031 & 0.032 & 0.032 \\
\hline & $z_{T P, l m, t}$ & 0.027 & 0.026 & 0.025 & 0.025 & 0.025 & 0.025 \\
\hline & $z_{T P, r m, t}$ & 0.024 & 0.022 & 0.021 & 0.021 & 0.022 & 0.022 \\
\hline \multirow[t]{3}{*}{3 minutes } & $z_{T P, t}$ & 0.044 & 0.045 & 0.043 & 0.044 & 0.044 & 0.044 \\
\hline & $z_{T P, l m, t}$ & 0.032 & 0.031 & 0.031 & 0.031 & 0.030 & 0.030 \\
\hline & $z_{T P, r m, t}$ & 0.025 & 0.024 & 0.024 & 0.024 & 0.024 & 0.023 \\
\hline \multirow[t]{3}{*}{5 minutes } & $z_{T P, t}$ & 0.053 & 0.051 & 0.052 & 0.051 & 0.051 & 0.050 \\
\hline & $z_{T P, l m, t}$ & 0.035 & 0.033 & 0.033 & 0.032 & 0.032 & 0.032 \\
\hline & $z_{T P, r m, t}$ & 0.025 & 0.023 & 0.023 & 0.023 & 0.023 & 0.023 \\
\hline \multirow[t]{3}{*}{30 minutes } & $z_{T P, t}$ & 0.150 & 0.151 & 0.150 & 0.151 & 0.151 & 0.150 \\
\hline & $z_{T P, l m, t}$ & 0.074 & 0.074 & 0.073 & 0.073 & 0.072 & 0.073 \\
\hline & $z_{T P, r m, t}$ & 0.029 & 0.029 & 0.029 & 0.029 & 0.028 & 0.029 \\
\hline
\end{tabular}

Fixed parameters: level of significance $=0.010, \alpha_{v}=-0.100, \sigma_{j m p}=1.50, \lambda=0.014$. 
Table 14 Confusion matrices, large rare jumps, and market microstructure noise $\left(z_{T P, r e n, t}\right)$.

\begin{tabular}{|c|c|c|c|c|c|c|c|c|c|}
\hline \multirow[b]{3}{*}{ Interval } & & \multicolumn{8}{|c|}{$\sigma_{m n}$} \\
\hline & & \multicolumn{2}{|c|}{0.000} & \multicolumn{2}{|c|}{0.027} & \multicolumn{2}{|c|}{0.052} & \multicolumn{2}{|c|}{0.080} \\
\hline & & $(\mathrm{NJ})$ & (J) & $(\mathrm{NJ})$ & (J) & $(\mathrm{NJ})$ & (J) & $(\mathrm{NJ})$ & (J) \\
\hline \multicolumn{10}{|c|}{$(i=0)$} \\
\hline \multirow[t]{2}{*}{1 minute } & $(\mathrm{NJ})$ & 0.988 & 0.012 & 0.996 & 0.004 & 1.000 & 0.000 & 1.000 & 0.000 \\
\hline & $(\mathrm{J})$ & 0.214 & 0.786 & 0.260 & 0.740 & 0.393 & 0.607 & 0.558 & 0.442 \\
\hline \multirow[t]{2}{*}{3 minutes } & $(\mathrm{NJ})$ & 0.988 & 0.012 & 0.989 & 0.011 & 0.995 & 0.005 & 0.998 & 0.002 \\
\hline & $(\mathrm{J})$ & 0.292 & 0.708 & 0.308 & 0.692 & 0.377 & 0.623 & 0.461 & 0.539 \\
\hline \multirow[t]{2}{*}{5 minutes } & $(\mathrm{NJ})$ & 0.986 & 0.014 & 0.986 & 0.014 & 0.991 & 0.009 & 0.995 & 0.005 \\
\hline & $(\mathrm{J})$ & 0.360 & 0.640 & 0.354 & 0.646 & 0.396 & 0.604 & 0.503 & 0.497 \\
\hline \multirow[t]{3}{*}{30 minutes } & $(\mathrm{NJ})$ & 0.986 & 0.014 & 0.985 & 0.015 & 0.985 & 0.015 & 0.986 & 0.014 \\
\hline & $(\mathrm{J})$ & 0.744 & 0.257 & 0.724 & 0.276 & 0.734 & 0.266 & 0.740 & 0.260 \\
\hline & & & & $(i=1$ & & & & & \\
\hline \multirow[t]{2}{*}{1 minute } & $(\mathrm{NJ})$ & 0.988 & 0.012 & 0.989 & 0.011 & 0.988 & 0.012 & 0.988 & 0.012 \\
\hline & $(\mathrm{J})$ & 0.224 & 0.776 & 0.253 & 0.747 & 0.321 & 0.679 & 0.432 & 0.568 \\
\hline \multirow[t]{2}{*}{3 minutes } & $(\mathrm{NJ})$ & 0.986 & 0.014 & 0.986 & 0.014 & 0.987 & 0.013 & 0.988 & 0.012 \\
\hline & $(\mathrm{J})$ & 0.286 & 0.714 & 0.305 & 0.695 & 0.341 & 0.659 & 0.416 & 0.584 \\
\hline \multirow[t]{2}{*}{5 minutes } & $(\mathrm{NJ})$ & 0.986 & 0.014 & 0.986 & 0.014 & 0.986 & 0.014 & 0.986 & 0.014 \\
\hline & $(\mathrm{J})$ & 0.344 & 0.656 & 0.370 & 0.630 & 0.422 & 0.578 & 0.481 & 0.519 \\
\hline \multirow[t]{3}{*}{30 minutes } & $(\mathrm{NJ})$ & 0.980 & 0.020 & 0.980 & 0.020 & 0.980 & 0.020 & 0.981 & 0.019 \\
\hline & $(\mathrm{J})$ & 0.708 & 0.292 & 0.721 & 0.279 & 0.718 & 0.282 & 0.750 & 0.250 \\
\hline & & & & $(i=2$ & & & & & \\
\hline \multirow[t]{2}{*}{1 minute } & $(\mathrm{NJ})$ & 0.988 & 0.012 & 0.989 & 0.011 & 0.988 & 0.012 & 0.987 & 0.013 \\
\hline & (J) & 0.221 & 0.779 & 0.266 & 0.734 & 0.331 & 0.669 & 0.409 & 0.591 \\
\hline \multirow[t]{2}{*}{3 minutes } & $(\mathrm{NJ})$ & 0.985 & 0.015 & 0.986 & 0.014 & 0.985 & 0.015 & 0.985 & 0.015 \\
\hline & $(\mathrm{J})$ & 0.286 & 0.714 & 0.325 & 0.675 & 0.383 & 0.617 & 0.442 & 0.558 \\
\hline \multirow[t]{2}{*}{5 minutes } & $(\mathrm{NJ})$ & 0.984 & 0.016 & 0.986 & 0.014 & 0.985 & 0.015 & 0.985 & 0.015 \\
\hline & $(\mathrm{J})$ & 0.360 & 0.640 & 0.390 & 0.610 & 0.435 & 0.565 & 0.490 & 0.510 \\
\hline \multirow[t]{2}{*}{30 minutes } & $(\mathrm{NJ})$ & 0.975 & 0.025 & 0.974 & 0.026 & 0.975 & 0.025 & 0.974 & 0.026 \\
\hline & $(\mathrm{J})$ & 0.737 & 0.263 & 0.730 & 0.270 & 0.737 & 0.263 & 0.750 & 0.250 \\
\hline
\end{tabular}

Fixed parameters: level of significance $=0.010, \alpha_{v}=-0.100, \sigma_{j m p}=1.50, \lambda=0.014$.

maximum adjustment in the estimate of scale, the size and power properties are excellent, and the test statistics appear to do an outstanding job of identifying the days on which jumps occur. Under modeling assumptions that include Gaussian microstructure noise, the effect of market microstructure noise is to bias the tests against finding jumps. That bias can be corrected using Andersen, Bollerslev, and Diebold's (2004) technique of staggering returns sufficiently to diminish the effects of the local serial correlation induced by microstructure noise. Although any Monte Carlo study is necessarily limited, and the theoretical work is constrained by the assumptions, it nonetheless seems reasonable to make valid 
Table 15 Proportion of days identified as jumps by the daily statistics.

\begin{tabular}{|c|c|c|c|c|c|}
\hline Level of significance & 0.500 & 0.950 & 0.990 & 0.995 & 0.999 \\
\hline \multicolumn{6}{|c|}{ S\&P 500 index futures $(04 / 21 / 1982-04 / 18 / 1997)$} \\
\hline \multicolumn{6}{|c|}{$(i=0)$} \\
\hline$z_{T P, t}$ & 0.64384 & 0.20941 & 0.11925 & 0.09651 & 0.06293 \\
\hline$z_{T P, l m, t}$ & 0.64384 & 0.17980 & 0.08699 & 0.06716 & 0.04019 \\
\hline$z_{T P, r m, t}$ & 0.64384 & $\begin{array}{c}0.15732 \\
(i=1)\end{array}$ & 0.06346 & 0.04469 & 0.02327 \\
\hline$z_{T P, t}$ & 0.68112 & 0.25754 & 0.15415 & 0.12850 & 0.08726 \\
\hline$z_{T P, l m, t}$ & 0.68112 & 0.22263 & 0.11687 & 0.09228 & 0.05500 \\
\hline$z_{T P, r m, t}$ & 0.68112 & $\begin{array}{c}0.19751 \\
(i=2)\end{array}$ & 0.08858 & 0.06319 & 0.03411 \\
\hline$z_{T P, t}$ & 0.71549 & 0.29799 & 0.18879 & 0.16288 & 0.11528 \\
\hline$z_{T P, l m, t}$ & 0.71549 & 0.25939 & 0.14728 & 0.11819 & 0.07377 \\
\hline$z_{T P, r m, t}$ & 0.71549 & 0.23559 & 0.11396 & 0.08726 & 0.04707 \\
\hline \multicolumn{6}{|c|}{$\begin{array}{c}(04 / 21 / 1997-10 / 22 / 2002) \\
(i=0)\end{array}$} \\
\hline$z_{T P, t}$ & 0.66108 & 0.20918 & 0.11152 & 0.09402 & 0.05175 \\
\hline$z_{T P, l m, t}$ & 0.66108 & 0.17566 & 0.07726 & 0.05831 & 0.03061 \\
\hline$z_{T P, r m, t}$ & 0.66108 & $\begin{array}{c}0.14942 \\
(i=1)\end{array}$ & 0.05758 & 0.03499 & 0.01603 \\
\hline$z_{T P, t}$ & 0.62026 & 0.20408 & 0.11953 & 0.09184 & 0.06195 \\
\hline$z_{T P, l m, t}$ & 0.62026 & 0.17055 & 0.08163 & 0.06341 & 0.03134 \\
\hline$z_{T P, r m, t}$ & 0.62026 & $\begin{array}{c}0.15452 \\
(i=2)\end{array}$ & 0.05977 & 0.03790 & 0.02332 \\
\hline$z_{T P, t}$ & 0.60714 & 0.19096 & 0.11006 & 0.09111 & 0.06706 \\
\hline$z_{T P, l m, t}$ & 0.60714 & 0.16327 & 0.08309 & 0.06924 & 0.04519 \\
\hline$\underline{z_{T P, r m, t}}$ & 0.60714 & 0.14213 & 0.06560 & 0.05248 & 0.02551 \\
\hline \multicolumn{6}{|c|}{$\begin{array}{c}(04 / 21 / 1982-12 / 09 / 2002) \\
(i=0)\end{array}$} \\
\hline$z_{T P, t}$ & 0.64886 & 0.20960 & 0.11705 & 0.09583 & 0.05997 \\
\hline$z_{T P, l m, t}$ & 0.64886 & 0.17875 & 0.08427 & 0.06479 & 0.03779 \\
\hline$z_{T P, r m, t}$ & 0.64886 & $\begin{array}{r}0.15523 \\
(i=1)\end{array}$ & 0.06190 & 0.04223 & 0.02140 \\
\hline$z_{T P, t}$ & 0.66506 & 0.24277 & 0.14462 & 0.11859 & 0.08060 \\
\hline$z_{T P, l m, t}$ & 0.66506 & 0.20845 & 0.10740 & 0.08465 & 0.04859 \\
\hline$z_{T P, r m, t}$ & 0.66506 & $\begin{array}{r}0.18569 \\
(i=2)\end{array}$ & 0.08099 & 0.05650 & 0.03105 \\
\hline$z_{T P, t}$ & 0.68608 & 0.26938 & 0.16776 & 0.14366 & 0.10220 \\
\hline$z_{T P, l m, t}$ & 0.68608 & 0.23351 & 0.13016 & 0.10490 & 0.06595 \\
\hline$z_{T P, r m, t}$ & 0.68608 & 0.21037 & 0.10085 & 0.07790 & 0.04126 \\
\hline
\end{tabular}


Table 16 Summary of $R V, B V$, and $R J$.

\begin{tabular}{|c|c|c|c|c|}
\hline Statistics & $R V$ & $B V$ & $R V-B V$ & $R J$ \\
\hline \multicolumn{5}{|c|}{$\begin{array}{l}\text { S\&P } 500 \text { index futures }(04 / 21 / 1982-04 / 18 / 1997) \\
\qquad(i=0)\end{array}$} \\
\hline Sample mean & 1.45438 & 1.25291 & 0.20146 & 0.04601 \\
\hline SD of mean & 0.28803 & 0.16804 & 0.12000 & 0.00182 \\
\hline \multicolumn{5}{|c|}{$(i=1)$} \\
\hline Sample mean & 1.45438 & 1.26816 & 0.18622 & 0.06017 \\
\hline SD of mean & 0.28803 & 0.20411 & 0.08393 & 0.00189 \\
\hline \multicolumn{5}{|c|}{$(i=2)$} \\
\hline Sample mean & 1.45438 & 1.24790 & 0.20647 & 0.07269 \\
\hline SD of mean & 0.28803 & 0.19699 & 0.09105 & 0.00198 \\
\hline Full-sample statistics & 5500.46066 & 4719.57255 & 780.88811 & 0.14197 \\
\hline \multicolumn{5}{|c|}{$\begin{array}{c}(04 / 21 / 1997-10 / 22 / 2002) \\
(i=0)\end{array}$} \\
\hline Sample mean & 2.10718 & 2.01193 & 0.09525 & 0.04445 \\
\hline SD of mean & 0.06690 & 0.06266 & 0.00424 & 0.00279 \\
\hline Full-sample statistics & 2891.05330 & 2760.37224 & 130.68106 & 0.04520 \\
\hline \multicolumn{5}{|c|}{$(i=1)$} \\
\hline Sample mean & 2.10718 & 2.01341 & 0.09378 & 0.04095 \\
\hline SD of mean & 0.06690 & 0.06133 & 0.00557 & 0.00302 \\
\hline Full-sample statistics & 2891.05330 & 2762.39293 & 128.66038 & 0.04450 \\
\hline \multicolumn{5}{|c|}{$(i=2)$} \\
\hline Sample mean & 2.10718 & 2.02129 & 0.08589 & 0.03556 \\
\hline SD of mean & 0.06690 & 0.06211 & 0.00479 & 0.00311 \\
\hline Full-sample statistics & 2891.05330 & 2773.21121 & 177.84209 & 0.04076 \\
\hline \multicolumn{5}{|c|}{$\begin{array}{c}(04 / 21 / 1982-12 / 09 / 2002) \\
(i=0)\end{array}$} \\
\hline Sample mean & 1.63393 & 1.46074 & 0.17319 & 0.04562 \\
\hline $\mathrm{SD}$ of mean & 0.21083 & 0.12375 & 0.08708 & 0.00152 \\
\hline Full-sample statistics & 8473.55466 & 7575.38272 & 898.17194 & 0.10600 \\
\hline \multicolumn{5}{|c|}{$(i=1)$} \\
\hline Sample mean & 1.63393 & 1.47225 & 0.16168 & 0.05500 \\
\hline SD of mean & 0.21083 & 0.14980 & 0.06103 & 0.00160 \\
\hline Full-sample statistics & 8473.55466 & 7635.08331 & 838.47135 & 0.09895 \\
\hline \multicolumn{5}{|c|}{$(i=2)$} \\
\hline Sample mean & 1.63393 & 1.45973 & 0.17420 & 0.06266 \\
\hline SD of mean & 0.21083 & 0.14467 & 0.06616 & 0.00168 \\
\hline Full-sample statistics & 8473.55466 & 7570.17627 & 903.37839 & 0.10661 \\
\hline
\end{tabular}

nonparametric claims for the reliability of the tests. That claim could be further buttressed (or possibly discredited) by additional research using more complicated data generating processes such as those with regime shifts or jumps in volatility. 
A good starting point for the latter would be the pure jump nonnegative OrnsteinUhlenbeck (OU) processes introduced in Barndorff-Nielsen and Shephard (2001), with generalizations reviewed and simulation schemes presented in Todorov and Tauchen (2004). Another avenue to strengthen the nonparametric claim would be to check whether the jump tests can uncover possible serial correlation in jumps, as suggested by evidence in Andersen, Bollerslev, and Huang (2005).

The Monte Carlo work also suggests that the new test statistics, while powerful, are not consistent tests. We believe that only by computing the statistics over a full sample, instead of day by day, can a consistent test be developed. However, our Monte Carlo work identifies a serious pitfall in this effort. Successful development requires a nonparametric way of knocking out a small sample bias that otherwise gets greatly magnified when summed over many days. We defer this task to future work.

The empirical work indicates strong evidence for jumps, where jumps account for about $4.5 \%$ to $7.0 \%$ of the total daily variance of the S\&P index, cash or futures. Interestingly, that evidence is obtained with a test likely biased away from finding jumps, and redoing the tests with the adjustment for microstructure noise raises the test statistics somewhat. The case for jumps thus appears compelling. The presence of jumps perforce precludes the prevalent assumption of local continuity and suggests that financial econometricians will need to enrich considerably the class of parametric models used to model very high frequency price series. The recent book by Cont and Tankov (2004) provides a powerful toolkit for creating more elaborate models. Although parametric modeling of very high frequency time series with jumps presents an intriguing statistical challenge, there is the serious issue of how economically important is the relatively small average contribution of $4.5 \%$ to $7.0 \%$ of total daily price variance. That question can only be addressed by examining the portfolio optimization behavior of an economic agent facing price series generated by the enhanced statistical models just discussed.

Received April 1, 2004; revised March 17, 2005; accepted July 4, 2005

\section{REFERENCES}

Ait-Sahalia, Y. (2002). "Telling From Discrete Data Whether the Underlying Continuous-Time Model is a Diffusion." Journal of Finance 57, 2075-2112.

Ait-Sahalia, Y. (2004). "Disentangling Diffusion from Jumps." Journal of Financial Economics 74, 487-528.

Aït-Sahalia, Y., P. Mykland, and L. Zhang. (2005). "How Often to Sample a Continuous-Time Process in the Presence of Market Microstructure Noise." Review of Financial Studies 18, 351-416.

Alizadeh, S., M. W. Brandt, and F. X. Diebold. (2002). "Range-Based Estimation of Stochastic Volatility Models." Journal of Finance 57, 1047-1091.

Andersen, T. G., L. Benzoni, and J. Lund. (2002). "An Empirical Investigation of Continuous-Time Equity Return Models." Journal of Finance 57, 1239-1284.

Andersen, T. G., T. Bollerslev, and F. X. Diebold. (2002). "Parametric and Nonparametric Volatility Measurement." In Y. Ait-Sahalia and L. P. Hansen (eds.), Handbook of Financial Econometrics. Amsterdam: North-Holland. 
Andersen, T. G., T. Bollerslev, and F. X. Diebold. (2004). "Some Like it Smooth, and Some Like it Rough: Untangling Continuous and Jump Components in Measuring, Modeling and Forecasting Asset Return Volatility." Working paper, Duke University.

Andersen, T. G., T. Bollerslev, F. X. Diebold, and H. Ebens. (2001a). "The Distribution of Realized Stock Return Volatility." Journal of Financial Economics, 61, 43-76.

Andersen, T. G., T. Bollerslev, F. X. Diebold, and P. Labys. (2001b). "The Distribution of Realized Exchange Rate Volatility." Journal of the American Statistical Association $96,42-55$.

Andersen, T. G., T. Bollerslev, F. X. Diebold, and P. Labys. (2003). "Modeling and Forecasting Realized Volatility." Econometrica 71, 579-625.

Andersen, T. G., T. Bollerslev, and X. Huang. (2005). "A Semiparametric Framework for Modelling and Forecasting Jumps and Volatility in Speculative Prices." Working paper, Duke University.

Bandi, F. M., and J. R. Russell. (2005a). "Microstructure Noise, Realized Variance, and Optimal Sampling." Working paper, University of Chicago.

Bandi, F. M., and J. R. Russell. (2005b). "Separating Microstructure Noise from Volatility." Working paper, University of Chicago; forthcoming in Journal of Financial Economics.

Barndorff-Nielsen, O. E., S. E. Graversen, J. Jacod, M. Podolskij, and N. Shephard. (2005). "A Central Limit Theorem for Realised Power and Bipower Variations of Continuous Semimartingales." Working paper, Nuffield College, Oxford University; forthcoming in Y. Kabanov and R. Liptser (eds.), From Stochastic Analysis to Mathematical Finance, Festschrift for Albert Shiryaev. New York: Springer-Verlag.

Barndorff-Nielsen, O. E., S. E. Graversen, J. Jacod, and N. Shephard. (2005). "Limit Theorems for Bipower Variation in Financial Econometrics." Working paper, Nuffield College, Oxford University.

Barndorff-Nielsen, O. E., and N. Shephard. (2001). "Non-Gaussian Ornstein-UhlenbeckBased Models and Some of their Uses in Financial Economics." Journal of the Royal Statistical Society, Series B 63, 167-241.

Barndorff-Nielsen, O. E., and N. Shephard. (2004a). "Measuring the Impact of Jumps on Multivariate Price Processes Using Bipower Variation." Discussion paper, Nuffield College, Oxford University.

Barndorff-Nielsen, O. E., and N. Shephard. (2004b). "Power and Bipower Variation with Stochastic Volatility and Jumps." Journal of Financial Econometrics 2, 1-37.

Barndorff-Nielsen, O. E., and N. Shephard. (2005a). "How Accurate is the Asymptotic Approximation to the Distribution of Realized Volatility." Forthcoming in D. W. K. Andrews, J. L. Powell, P. A. Ruud, and J. H. Stock (eds.), Identification and Inference for Econometric Models. Essays in Honor of Thomas Rothenberg. Cambridge: Cambridge University Press.

Barndorff-Nielsen, O. E., and N. Shephard. (2005b). "Variation, Jumps, Market Frictions and High Frequency Data in Financial Econometrics." Discussion paper prepared for the 9th World Congress of the Econometric Society, Nuffield College, Oxford University.

Barndorff-Nielsen, O. E., and N. Shephard. (2006). “Econometrics of Testing for Jumps in Financial Economics Using Bipower Variation." Forthcoming in Journal of Financial Econometrics.

Basawa, I. V., and P. J. Brockwell. (1982). "Non-Parametric Estimation for NonDecreasing Lévy Processes." Journal of the Royal Statistical Society, Series B 44, 262-269. 
Chernov, M., A. R. Gallant, E. Ghysels, and G. Tauchen. (2003). “Alternative Models for Stock Price Dynamics." Journal of Econometrics 116, 225-257.

Clark, P. K. (1973). "A Subordinated Stochastic Process Model with Finite Variance for Speculative Prices." Econometrica 41, 135-155.

Conrad, J., G. Kaul, and M. Nimalendran. (1991). "Components of Short-Horizon Individual Security Returns." Journal of Financial Economics 29, 365-384.

Cont, R. and P. Tankov. (2004). Financial Modelling with Jump Processes. Boca Raton, FL: Chapman \& Hall/CRC.

Corradi, V., and W. Distaso. (2004). "Estimating and Testing Stochastic Volatility Models Using Realized Measures." Working paper, University of London.

Duffie, D., J. Pan, and K. Singleton. (2000). "Transform Analysis and Asset Pricing for Affine Jump-Diffusions." Econometrica 68, 1343-1376.

Eraker, B., M. S. Johannes, and N. G. Polson. (2003). “The Impact of Jumps in Volatility and Returns." Journal of Finance 58, 1269-1300.

Gallant, A. R., C.-T. Hsu, and G. Tauchen. (1999). "Using Daily Range Data to Calibrate Volatility Diffusions and Extract the Forward Integrated Variance." Review of Economics and Statistics 81, 617-631.

Gonçalves, S., and N. Meddahi. (2005). “Bootstrapping Realized Volatility.” Working paper, Université de Montréal.

Hansen, P. R., and A. Lunde. (2004a). "Realized Variance and Market Microstructure Noise." Working paper, Stanford University.

Hansen, P. R., and A. Lunde. (2004b). "An Unbiased Measure of Realized Variance." Working paper, Stanford University.

Hasbrouck, J. (1993). "Assessing the Quality of a Security Market: A New Approach to Transaction-Cost Measurement." Review of Financial Studies 6, 191-212.

Hausman, J. A. (1978). "Specification Tests in Econometrics." Econometrica 46, 1251-1271.

Kaul, G., and M. Nimalendran. (1990). "Price Reversals: Bid-Ask Errors or Market Overreaction?" Journal of Financial Economics 28, 67-93.

Kloeden, P. E., and E. Platen. (1992). Numerical Solution of Stochastic Differential Equations. New York: Springer-Verlag.

Madhavan, A., M. Richardson, and M. Roomans. (1997). "Why do Security Prices Change? A Transaction-Level Analysis of NYSE Stocks." Review of Financial Studies 10, 1035-1064.

Merton, R. C. (1976). “Option Pricing When Underlying Stock Returns are Discontinuous." Journal of Financial Economics 3, 125-144.

Shephard, N. (2005). Stochastic Volatility: Selected Readings. Oxford: Oxford University Press.

Taylor, S. J. (1982). "Financial Returns Modelled by the Product of Two Stochastic Processes, a Study of Daily Sugar Prices, 1961-79. "In O. D. Anderson (ed.), Time Series Analysis: Theory and Practice 1. Amsterdam: North-Holland.

Taylor, S. J. (1986). Modelling Financial Time Series. Chichester: John Wiley \& Sons.

Todorov, V., and G. Tauchen. (2004). "Simulation Methods for Lévy-Driven CARMA Stochastic Volatility Models." Working paper, Duke University.

Zhang, L., P. Mykland, and Y. Aït-Sahalia. (2004). "A Tale of Two Time Scales: Determining Integrated Volatility with Noisy High-Frequency Data." Forthcoming in Journal of the American Statistical Association. 
Reproduced with permission of the copyright owner. Further reproduction prohibited without permission. 